\title{
A mixed-primal finite element method for the coupling of Brinkman-Darcy flow and nonlinear transport $\dagger$
}

\author{
MARio Alvarez $\ddagger$ \\ Sección de Matemática, Sede de Occidente, Universidad de Costa Rica, San Ramón de \\ Alajuela, Costa Rica \\ GABRIEL N. GATICA $\S$ \\ $C I^{2} M A$, Universidad de Concepción, Casilla 160-C, Concepción, Chile; and \\ Departamento de Ingeniería Matemática, Universidad de Concepción, Chile \\ AND \\ RICARDO RUIZ-BAIER $₫$ \\ Mathematical Institute, University of Oxford, Woodstock Road, OX2 6GG Oxford, UK; and \\ Universidad Adventista de Chile, Casilla 7-D, Chillán, Chile
}

[Received on 22 April 2019; revised on 19 September 2019; accepted on 5 November 2019]

\begin{abstract}
This paper is devoted to the mathematical and numerical analysis of a model describing the interfacial flow-transport interaction in a porous-fluidic domain. The medium consists of a highly permeable material, where the flow of an incompressible viscous fluid is governed by Brinkman equations (written in terms of vorticity, velocity and pressure), and a porous medium where Darcy's law describes fluid motion using filtration velocity and pressure. Gravity and the local fluctuations of a scalar field (representing for instance, the solids volume fraction, or the concentration of a contaminant) are the main drivers of the fluid patterns on the whole domain, and the Brinkman-Darcy equations are coupled to a nonlinear transport equation accounting for mass balance of the scalar concentration. We introduce a mixed-primal variational formulation of the problem and establish existence and uniqueness of solution using fixed-point arguments and small-data assumptions. A family of Galerkin discretisations that produce divergence-free discrete velocities is also presented and analysed using similar tools to those employed in the continuous problem. Convergence of the resulting mixed-primal finite element method is proven, and some numerical examples confirming the theoretical error bounds and illustrating the performance of the proposed discrete scheme are reported.
\end{abstract}

Keywords: Nonlinear transport, Brinkman-Darcy coupling, vorticity-based formulation, fixed-point theory, mixed finite elements, error analysis.

Mathematical subject classification 2000: . 65N30, 76S05, 65N12, 65N15.

\section{Introduction}

The aim of this paper is to put together an extension of the results from Alvarez et al. (2015 2016a) and Alvarez et al. (2016b) dealing with augmented and fully-mixed finite element approximations of coupled flow and transport problems, and coupled Brinkman and Darcy flow, respectively. The coupled system describes the interaction of flow and transport phenomena in two different domains separated by an interface. Such a formalism arises naturally, and has been systematically used, in hydrology and biological applications including

\footnotetext{
${ }^{\dagger}$ Funding: This research was partially supported by Vicerrectoría de Investigación, project 540-B7-233, Sede de Occidente, Universidad de Costa Rica; by CONICYT-Chile through project AFB170001 of the PIA Program: Concurso Apoyo a Centros Científicos y Tecnológicos de Excelencia con Financiamiento Basal; by Centro de Investigación en Ingeniería Matemática ( $\left.\mathrm{CI}^{2} \mathrm{MA}\right)$, Universidad de Concepción; and by the EPSRC through the Research Grant EP/R00207X/1.

${ }^{\ddagger}$ Email: mario.alvarezguadamuz@ucr.ac.cr

§Email: ggatica@ci2ma.udec.cl

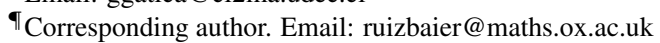


for instance subsurface flow, hydraulic fractures, $\mathrm{CO}_{2}$ sequestration, perfusion of soft living tissues, etc. In obtaining approximate solutions for the problem under consideration one faces marked difficulties. These are related to the coupling of mechanisms that act simultaneously, such as active transport and reaction of the solute and nonlinearities in the diffusion process and in the source term; as well as the heterogeneities, transmission conditions, and the need of preserving physical properties. Even if many numerical solutions are already available (see e.g. Ehrhardt et al. (2009); Jena et al. (2013); Joodi et al. (2010); Khaled \& Vafai) (2003) and the references therein), up to the authors' knowledge the only contributions addressing mathematical and numerical properties of somewhat similar couplings are the recent works Çeşmelioğlu et al. (2012), where existence and stability bounds of weak solutions is established also for the nonlinear Navier-Stokes-Darcy flow coupled with transport; Vassilev \& Yotov (2009), where a mixed finite element scheme approximates the Stokes-Darcy system and a local discontinuous Galerkin method is employed to discretise the transport equation; Rui \& Zhang (2017) that analyses stabilised velocity-pressure-concentration formulations for a model where viscosity depends on the solute concentration; and the very recent works Ervin et al. (2019); Zhang et al. (2019) focusing on discrete analysis of splitting of time stepping between the conforming discretisations on the two subdomains.

The main difference of these works with respect to our contribution, is that we propose a formulation of the problem written in terms of Brinkman vorticity, and the transmission conditions we employ are slightly different. In addition, we introduce a mixed-primal finite element method for the Brinkman-Darcy-Transport coupling that produces divergence-free discrete velocities. Another clear motivation for using a vorticity-based formulation in the Brinkman domain is that the method is pressure-robust, it computes the vorticity vector directly and avoiding derivative-based postprocessing (this field is of utmost importance in determining properties for a variety of regimes of incompressible fluids, e.g. in subsurface flow or blood flow, or other applications where the patterns of rotational flow are of interest Pontrelli (2001); Riaz et al. (2006); Speziale (1987)), and is of relatively low computational cost when compared to other mixed formulations using for instance vorticity tensors. These features are essentially inherited from other schemes such as the methods for Stokes or Brinkman equations advanced in Anaya et al. (2013. 2016); Vassilevski \& Villa (2014).

Following our previous work Alvarez et al. (2016b), the coupling of subdomains is based on a vorticity based fully-mixed formulation for the Brinkman-Darcy problem, whereas a primal formulation for the transport problem is adapted from Alvarez et al. (2016a). The solvability of such a coupling will be based on extending the fixed-point strategy introduced in Alvarez et al. (2015) and Alvarez et al. (2016a) to the present context. In particular, we realise that the primal formulation for the transport problem requires further regularity for the global velocity, initially living in $\mathbf{H}(\operatorname{div}, \Omega)$. In turn, and in contrast with Alvarez et al. (2015) and Alvarez et al. (2016a), we can not exploit augmentation techniques to recover $\mathbf{H}^{1}(\Omega)$ velocities. Instead, a different smoothness assumption is introduced at the level of the continuous analysis of the transport problem, and subsequently in the solvability of the Brinkman-Darcy-Transport coupling. More precisely, the derivation of existence of weak solutions relies on a strategy combining classical fixed-point arguments, suitable regularity assumptions on the decoupled problems, the Lax-Milgram Lemma, preliminary results from Alvarez et al. (2016b), and the Sobolev embedding and Rellich-Kondrachov compactness theorems. In addition, sufficiently small data allow us to establish uniqueness of weak solution. On the other hand, the well-posedness of the discrete problem is based on the Brouwer fixed-point theorem and analogous arguments to those employed in the continuous analysis. Finally, similar arguments as those utilised in Alvarez et al. (2016ab) allow us to derive the corresponding Céa estimates for both the Brinkman-Darcy and transport problems, and these lead to natural a priori error bounds for the Galerkin scheme.

OUTLINE. This paper has been structured as follows. The remainder of this section presents some notation and preliminary definitions of spaces needed thereafter. The model problem along with boundary data are stated in Section 2 The weak formulation of the problem and its well-posedness analysis in the framework of the Schauder fixed-point theorem are collected in Section 3 The associated Galerkin scheme is then proposed in Section 4 and its solvability is established by the Brouwer fixed-point theorem. Next, we derive in Section 5 some a priori error estimates, and conclude in Section 6 with a few numerical examples in 2D and $3 \mathrm{D}$, illustrating the good performance of the mixed-primal finite element method and confirming the expected error decay.

PreliminARIES. Standard notation will be adopted for Lebesgue and Sobolev spaces. In addition, by M and $\mathbb{M}$ we will denote the corresponding vectorial and tensorial counterparts of the generic scalar functional space $\mathrm{M}$, and $\|\cdot\|$, with no subscripts, will stand for the natural norm of either an element or an operator in any product functional space. For instance, if $\Theta \subseteq \mathrm{R}^{n}, n=2,3$ is a domain, $\Lambda \subseteq \mathrm{R}^{n}$ is a Lipschitz surface, and 
$r \in \mathrm{R}$, we define $\mathbf{H}^{r}(\Theta):=\left[\mathrm{H}^{r}(\Theta)\right]^{n}$ and $\mathbf{H}^{r}(\Lambda):=\left[\mathrm{H}^{r}(\Lambda)\right]^{n}$. We also recall the definition of the following Hilbert spaces

$$
\mathbf{H}(\operatorname{div} ; \Theta):=\left\{\boldsymbol{v} \in \mathbf{L}^{2}(\Theta): \quad \operatorname{div} \boldsymbol{v} \in \mathrm{L}^{2}(\Theta)\right\}, \quad \mathbf{H}(\operatorname{curl} ; \Theta):=\left\{\boldsymbol{v} \in \mathbf{L}^{2}(\Theta): \quad \operatorname{curl} \boldsymbol{v} \in \mathbf{L}^{2}(\Theta)\right\},
$$

normed, respectively, with

$$
\|\boldsymbol{v}\|_{\operatorname{div} ; \Theta}:=\left\{\|\boldsymbol{v}\|_{0, \Theta}^{2}+\|\operatorname{div} \boldsymbol{v}\|_{0, \Theta}^{2}\right\}^{1 / 2}, \quad\|\boldsymbol{v}\|_{\operatorname{curl} ; \Theta}:=\left\{\|\boldsymbol{v}\|_{0, \Theta}^{2}+\|\operatorname{curl} \boldsymbol{v}\|_{0, \Theta}^{2}\right\}^{1 / 2},
$$

where, for any vector field $v:=\left(v_{1}, \ldots, v_{d}\right)^{\mathrm{t}} \in \mathbf{L}^{2}(\Theta)$,

$$
\operatorname{div} \boldsymbol{v}:=\sum_{i=1}^{n} \partial_{i} v_{i}, \quad \operatorname{curl} \boldsymbol{v}:=\nabla \times \boldsymbol{v}=\left(\begin{array}{l}
\partial_{2} v_{3}-\partial_{3} v_{2} \\
\partial_{3} v_{1}-\partial_{1} v_{3} \\
\partial_{1} v_{2}-\partial_{2} v_{1}
\end{array}\right) \text { if } n=3, \text { and curl } \boldsymbol{v}=\partial_{1} v_{2}-\partial_{2} v_{1} \text { if } n=2 .
$$

In addition, we also recall the orthogonal decomposition

$$
\mathrm{L}^{2}(\Theta)=\mathrm{L}_{0}^{2}(\Theta) \oplus P_{0}(\Theta),
$$

where $P_{0}(\Theta)$ is the space of constant functions on $\Theta$, and

$$
\mathrm{L}_{0}^{2}(\Theta)=P_{0}(\Theta)^{\perp}:=\left\{q \in \mathrm{L}^{2}(\Theta): \quad \int_{\Theta} q=0\right\} .
$$

Equivalently, each $q \in \mathrm{L}^{2}(\Theta)$ can be uniquely decomposed as $q=q_{0}+c$, with

$$
q_{0}:=q-\frac{1}{|\Theta|} \int_{\Theta} q \in \mathrm{L}_{0}^{2}(\Theta) \quad \text { and } \quad c:=\frac{1}{|\Theta|} \int_{\Theta} q \in \mathrm{R}
$$

where $L_{0}^{2}(\Theta)$ is endowed with the usual norm of $L^{2}(\Theta)$, and it is easy to see that there holds

$$
\|q\|_{0, \Theta}^{2}=\left\|q_{0}\right\|_{0, \Theta}^{2}+|\Theta| c^{2} .
$$

By $\mathbf{0}$ we will denote the generic null vector (including the null functional and operator), and we will denote by $C$ and $c$, with or without subscripts, bars, tildes or hats, generic constants independent of the discretisation parameters.

\section{Governing equations}

Let $\Omega \subset \mathrm{R}^{n}, n=2,3$, denote an heterogeneous porous domain composed of two regions: $\Omega_{\mathrm{B}}$, where the viscous flow patterns characterised by velocity $\boldsymbol{u}_{\mathrm{B}}$, vorticity $\boldsymbol{\omega}_{\mathrm{B}}$, and pressure $p_{\mathrm{B}}$ can be governed by the linear Brinkman equations; and $\Omega_{\mathrm{D}}$, where the flow of the immiscible fluid obeys to Darcy's law written in terms of velocity $\boldsymbol{u}_{\mathrm{D}}$ and pressure $p_{\mathrm{D}}$ in the porous domain. These subdomains are separated by an interface $\Sigma$, through which exchange of fluid velocities and pressures occurs. We also consider that a given scalar field $\phi$ (representing, for instance, the concentration of a chemical component, the fluid density, the temperature, or the volume fraction or saturation of a solid phase) is advected and diffused on the whole $\Omega$ according to the mass conservation principle (or energy conservation if the scalar field stands for e.g. temperature). The model problem can be summarised as follows:

(Brinkman)

$$
\left.\begin{array}{rl}
\mu \mathbb{K}_{\mathrm{B}}^{-1} \boldsymbol{u}_{\mathrm{B}}+\mu \operatorname{curl} \boldsymbol{\omega}_{\mathrm{B}}+\nabla p_{\mathrm{B}} & =\phi f_{\mathrm{B}} \\
\boldsymbol{\omega}_{\mathrm{B}}-\operatorname{curl} \boldsymbol{u}_{\mathrm{B}} & =\mathbf{0} \\
\operatorname{div} \boldsymbol{u}_{\mathrm{B}} & =0
\end{array}\right\} \text { in } \Omega_{\mathrm{B}}
$$

coupled with

(Darcy)

$$
\left.\begin{array}{rl}
\mu \mathbb{K}_{\mathrm{D}}^{-1} \boldsymbol{u}_{\mathrm{D}}+\nabla p_{\mathrm{D}} & =\phi \boldsymbol{f}_{\mathrm{D}} \\
\operatorname{div} \boldsymbol{u}_{\mathrm{D}} & =0
\end{array}\right\} \quad \text { in } \quad \Omega_{\mathrm{D}}
$$

and

$$
\text { (Transport) } \quad \beta \phi-\operatorname{div}\left(\vartheta(\phi) \nabla \phi-\phi \boldsymbol{u}-f_{\mathrm{bk}}(\phi) \boldsymbol{g}\right)=0 \text { in } \Omega,
$$

where $\mu>0$ is the constant viscosity of the fluid in the entire domain $\Omega$, the parameter $\beta$ is the porosity of the medium (assumed constant inside each subdomain, but possibly discontinuous across $\Sigma$ ). Notice that $\boldsymbol{u}$ in 2.3 refers to the global velocity field defined in both $\Omega_{\mathrm{B}}$ and $\Omega_{\mathrm{D}}$, that is $\boldsymbol{u}:=\mathbf{1}_{\Omega_{\mathrm{B}}} \boldsymbol{u}_{\mathrm{B}}+\mathbf{1}_{\Omega_{\mathrm{D}}} \boldsymbol{u}_{\mathrm{D}}$, where $\mathbf{1}_{\Omega_{\star}}$ is the 

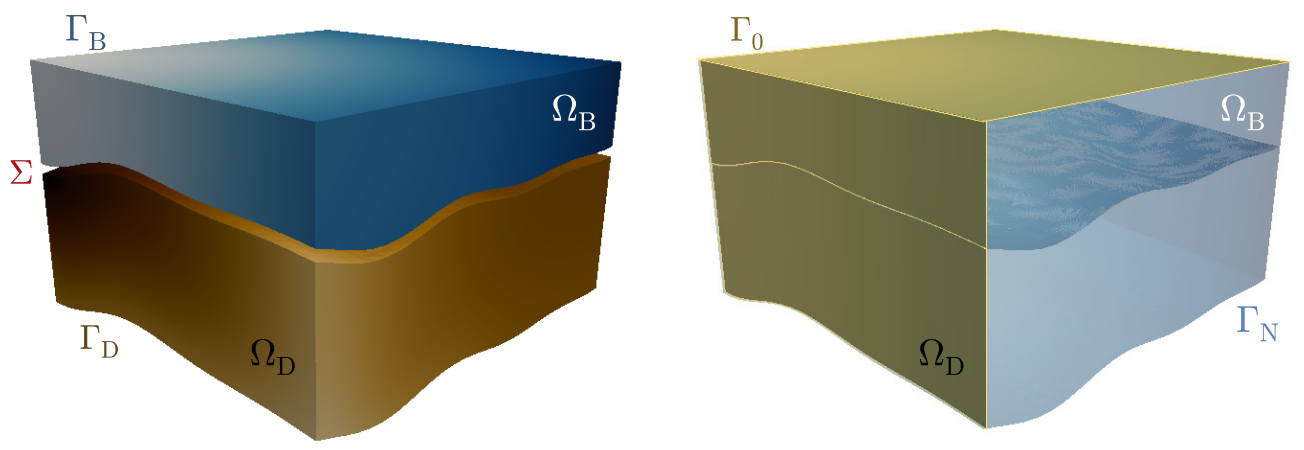

FIG. 2.1. Sketch of the domains occupied by the incompressible fluid and by the porous medium $\left(\Omega_{\mathrm{B}}\right.$ and $\Omega_{\mathrm{D}}$, respectively), interface $\Sigma$, and corresponding boundaries.

characteristic function, $\star \in\{B, D\}$. In addition, $\mathbb{K}_{B}$ and $\mathbb{K}_{D}$ are symmetric, bounded, and uniformly positive definite tensors $\mathbb{K}_{\mathrm{B}}, \mathbb{K}_{\mathrm{D}}$, which means that there exist $\alpha_{\mathbb{K}_{\mathrm{B}}}>0$ and $\alpha_{\mathbb{K}_{\mathrm{D}}}>0$ such that

$$
\boldsymbol{v}^{\mathrm{t}} \mathbb{K}_{\mathrm{B}}^{-1}(\boldsymbol{x}) \boldsymbol{v} \geqslant \alpha_{\mathbb{K}_{\mathrm{B}}}|\boldsymbol{v}|^{2} \quad \forall \boldsymbol{v} \in \mathrm{R}^{n}, \forall \boldsymbol{x} \in \Omega_{\mathrm{B}},
$$

and

$$
\boldsymbol{v}^{\mathrm{t}} \mathbb{K}_{\mathrm{D}}^{-1}(\boldsymbol{x}) \boldsymbol{v} \geqslant \alpha_{\mathbb{K}_{\mathrm{D}}}|\boldsymbol{v}|^{2} \quad \forall \boldsymbol{v} \in \mathrm{R}^{n}, \forall \boldsymbol{x} \in \Omega_{\mathrm{D}} .
$$

In turn, the tensors $\mathbb{K}_{\mathrm{B}}$ and $\mathbb{K}_{\mathrm{D}}$ characterise the absolute permeability of the Brinkman and Darcy domains, respectively; the function $\vartheta$ is a nonlinear diffusivity, and $f_{\mathrm{bk}}$ is a nonlinear flux acting on the direction of the gravity acceleration $\boldsymbol{g}$, aligned with the negative $x_{n}$-axis. The specific forms of these variable coefficients will be made precise later. In addition, we assume that $f_{\mathrm{B}} \in \mathbf{L}^{\infty}\left(\Omega_{\mathrm{B}}\right)$ and $f_{\mathrm{D}} \in \mathbf{L}^{\infty}\left(\Omega_{\mathrm{D}}\right)$. We stress that the local fluctuations of $\phi$ drive the flow patterns only through the external load in the momentum equations. In this sense, the coupling mechanisms considered here are somehow weaker than those studied in Alvarez et al. (2015 2016a) for transport-flow in a single domain (where also viscosity was depending of $\phi$ ).

We assume that $\Omega$ has a Lipschitz continuous boundary split into two disjoint sub-boundaries with positive measure, according to two criteria: firstly, $\partial \Omega=\Gamma_{\mathrm{B}} \cup \Gamma_{\mathrm{D}}$, where $\Gamma_{\mathrm{B}}=\partial \Omega_{\mathrm{B}} \backslash \Sigma$ and $\Gamma_{\mathrm{D}}=\partial \Omega_{\mathrm{D}} \backslash \Sigma$ denote pure Brinkman and Darcy borders, respectively; and secondly $\partial \Omega=\Gamma_{0} \cup \Gamma_{\mathrm{N}}$, where $\Gamma_{0}, \Gamma_{\mathrm{N}}$ denote the parts of the boundary where homogeneous Dirichlet or Neumann (zero flux) conditions are enforced for $\phi$, respectively (see a rough diagram of domains and boundaries in Figure 2.1). The considered boundary and transmission conditions are:

$$
\begin{gathered}
\boldsymbol{u}_{\mathrm{D}} \cdot \boldsymbol{n}=\boldsymbol{u}_{\mathrm{B}} \cdot \boldsymbol{n} \quad \text { and } \quad p_{\mathrm{D}}=p_{\mathrm{B}} \text { on } \Sigma, \\
\boldsymbol{\omega}_{\mathrm{B}} \times \boldsymbol{n}=0 \quad \text { on } \partial \Omega_{\mathrm{B}}=\Sigma \cup \Gamma_{\mathrm{B}}, \boldsymbol{u}_{\mathrm{B}} \cdot \boldsymbol{n}=0 \quad \text { on } \Gamma_{\mathrm{B}}, \quad \text { and } \boldsymbol{u}_{\mathrm{D}} \cdot \boldsymbol{n}=0 \quad \text { on } \Gamma_{\mathrm{D}}, \\
\phi=0 \quad \text { on } \Gamma_{0}, \quad \text { and }\left(\vartheta(\phi) \nabla \phi-\phi \boldsymbol{u}-f_{\mathrm{bk}}(\phi) \boldsymbol{g}\right) \cdot \boldsymbol{n}=0 \quad \text { on } \Gamma_{\mathrm{N}},
\end{gathered}
$$

where $\boldsymbol{n}$ denotes the outward normal at $\Omega_{\mathrm{B}}$ and $\Omega_{\mathrm{D}}$. Note that interface conditions are not required in the transport equation, as the continuity of $\phi$ and of the corresponding fluxes is incorporated naturally in the formulation.

For the sake of our analysis, the variable coefficients need to satisfy the following requirements: there exist positive constants $\vartheta_{1}, \vartheta_{2}, \gamma_{1}, \gamma_{2}, L_{\vartheta}$, and $L_{f_{\mathrm{bk}}}$, such that

$$
\begin{gathered}
\vartheta_{1} \leqslant \vartheta(s) \leqslant \vartheta_{2}, \quad \text { and } \quad \gamma_{1} \leqslant f_{\mathrm{bk}}(s) \leqslant \gamma_{2} \quad \forall s \in \mathrm{R}, \\
|\vartheta(s)-\vartheta(t)| \leqslant L_{\vartheta}|s-t| \quad \forall s, t \in \mathrm{R},
\end{gathered}
$$

and

$$
\left|f_{\mathrm{bk}}(s)-f_{\mathrm{bk}}(t)\right| \leqslant L_{f_{\mathrm{bk}}}|s-t| \quad \forall s, t \in \mathrm{R} .
$$

In view of deriving a weak form of (2.1)-(2.3), and according to the boundary data 2.4 , we introduce the following functional spaces

$$
\mathbf{H}_{\mathrm{B}}\left(\operatorname{div} ; \Omega_{\mathrm{B}}\right):=\left\{\boldsymbol{v}_{\mathrm{B}} \in \mathbf{H}\left(\operatorname{div} ; \Omega_{\mathrm{B}}\right): \quad \boldsymbol{v}_{\mathrm{B}} \cdot \boldsymbol{n}=0 \quad \text { on } \quad \Gamma_{\mathrm{B}}\right\},
$$




$$
\begin{aligned}
& \mathbf{H}_{0}\left(\operatorname{curl} ; \Omega_{\mathrm{B}}\right):=\left\{z_{\mathrm{B}} \in \mathbf{H}\left(\operatorname{curl} ; \Omega_{\mathrm{B}}\right): \quad z_{\mathrm{B}} \times \boldsymbol{n}=\mathbf{0} \quad \text { on } \quad \partial \Omega_{\mathrm{B}}\right\}, \\
& \mathbf{H}_{\mathrm{D}}\left(\operatorname{div} ; \Omega_{\mathrm{D}}\right):=\left\{\boldsymbol{v}_{\mathrm{D}} \in \mathbf{H}\left(\operatorname{div} ; \Omega_{\mathrm{D}}\right): \quad \boldsymbol{v}_{\mathrm{D}} \cdot \boldsymbol{n}=0 \quad \text { on } \Gamma_{\mathrm{D}}\right\},
\end{aligned}
$$

and

$$
\mathrm{H}_{\Gamma_{0}}^{1}(\Omega):=\left\{\psi \in \mathrm{H}^{1}(\Omega):\left.\psi\right|_{\Gamma_{0}}=0\right\},
$$

for which, thanks to the generalised Poincaré inequality, there exists $c_{p}>0$, depending only on $\Omega$ and $\Gamma_{0}$, such that

$$
\|\psi\|_{1, \Omega} \leqslant c_{p}|\psi|_{1, \Omega}, \quad \forall \psi \in \mathrm{H}_{\Gamma_{0}}^{1}(\Omega) .
$$

\section{Weak formulation and its solvability analysis}

In this section we proceed similarly as in Alvarez et al. (2015) and Alvarez et al. (2016a) to derive a suitable variational formulation of $2.1-(2.2)-(2.3)-(2.4)$ and analyse its solvability by means of a fixed-point strategy.

\subsection{Continuous mixed-primal formulation}

The continuity of pressure across the interface allows us to define its trace

$$
\lambda:=\left.p_{\mathrm{D}}\right|_{\Sigma}=\left.p_{\mathrm{B}}\right|_{\Sigma} \in \mathrm{H}^{1 / 2}(\Sigma) .
$$

Then, after testing the Brinkman momentum equation in 2.1 against $\boldsymbol{v}_{\mathrm{B}} \in \mathbf{H}_{\mathrm{B}}\left(\operatorname{div} ; \Omega_{\mathrm{B}}\right)$, and integrating by parts, we get

$$
\mu \int_{\Omega_{\mathrm{B}}} \mathbb{K}_{\mathrm{B}}^{-1} \boldsymbol{u}_{\mathrm{B}} \cdot \boldsymbol{v}_{\mathrm{B}}+\mu \int_{\Omega_{\mathrm{B}}} \boldsymbol{v}_{\mathrm{B}} \cdot \operatorname{curl} \boldsymbol{\omega}_{\mathrm{B}}-\int_{\Omega_{\mathrm{B}}} p_{\mathrm{B}} \operatorname{div} \boldsymbol{v}_{\mathrm{B}}+\left\langle\boldsymbol{v}_{\mathrm{B}} \cdot \boldsymbol{n}, \lambda\right\rangle_{\Sigma}=\int_{\Omega_{\mathrm{B}}} \phi \boldsymbol{f}_{\mathrm{B}} \cdot \boldsymbol{v}_{\mathrm{B}} \quad \forall \boldsymbol{v}_{\mathrm{B}} \in \mathbf{H}_{\mathrm{B}}\left(\operatorname{div} ; \Omega_{\mathrm{B}}\right) .
$$

Next, testing the constitutive equation in 2.1 against $z_{\mathrm{B}} \in \mathbf{H}_{0}\left(\mathbf{c u r l} ; \Omega_{\mathrm{B}}\right)$, and integrating by parts, we obtain

$$
\int_{\Omega_{\mathrm{B}}} \boldsymbol{\omega}_{\mathrm{B}} \cdot z_{\mathrm{B}}-\int_{\Omega_{\mathrm{B}}} u_{\mathrm{B}} \cdot \operatorname{curl} z_{\mathrm{B}}=0 \quad \forall z_{\mathrm{B}} \in \mathbf{H}_{0}\left(\operatorname{curl} ; \Omega_{\mathrm{B}}\right) .
$$

In turn, the incompressibility constraint in 2.1 in weak form reads

$$
\int_{\Omega_{\mathrm{B}}} q_{\mathrm{B}} \operatorname{div} \boldsymbol{u}_{\mathrm{B}}=0 \quad \forall q_{\mathrm{B}} \in \mathrm{L}^{2}\left(\Omega_{\mathrm{B}}\right) .
$$

On the other hand, testing the first equation of 2.2 with functions in $\mathbf{H}_{\mathrm{D}}\left(\operatorname{div} ; \Omega_{\mathrm{D}}\right)$, integrating by parts, using the boundary conditions, and employing 3.1 , we get

$$
\mu \int_{\Omega_{\mathrm{D}}} \mathbb{K}_{\mathrm{D}}^{-1} \boldsymbol{u}_{\mathrm{D}} \cdot \boldsymbol{v}_{\mathrm{D}}-\int_{\Omega_{\mathrm{D}}} p_{\mathrm{D}} \operatorname{div} \boldsymbol{v}_{\mathrm{D}}-\left\langle\boldsymbol{v}_{\mathrm{D}} \cdot \boldsymbol{n}, \lambda\right\rangle_{\Sigma}=\int_{\Omega_{\mathrm{D}}} \phi \boldsymbol{f}_{\mathrm{D}} \cdot \boldsymbol{v}_{\mathrm{D}} \quad \forall \boldsymbol{v}_{\mathrm{D}} \in \mathbf{H}_{\mathrm{D}}\left(\operatorname{div} ; \Omega_{\mathrm{D}}\right) .
$$

In addition, the second equation in 2.2 is tested as

$$
\int_{\Omega_{\mathrm{D}}} q_{\mathrm{D}} \operatorname{div} v_{\mathrm{D}}=0 \quad \forall q_{\mathrm{D}} \in \mathrm{L}^{2}\left(\Omega_{\mathrm{D}}\right)
$$

Finally, the continuity of normal velocities across $\Sigma$ ( $c f$. first equation in 2.4 ) is imposed weakly as follows

$$
\left\langle\boldsymbol{u}_{\mathrm{B}} \cdot \boldsymbol{n}-\boldsymbol{u}_{\mathrm{D}} \cdot \boldsymbol{n}, \boldsymbol{\xi}\right\rangle_{\Sigma}=0 \quad \forall \xi \in \mathrm{H}^{1 / 2}(\Sigma) .
$$

Therefore, given $\phi \in \mathrm{H}_{\Gamma_{0}}^{1}(\Omega)$, we arrive at the following mixed formulation for the Brinkman-Darcy coupling: Find $\overrightarrow{\boldsymbol{u}}:=\left(\boldsymbol{u}_{\mathrm{B}}, \boldsymbol{\omega}_{\mathrm{B}}, \boldsymbol{u}_{\mathrm{D}}\right) \in \mathbf{H}$ and $\vec{p}:=\left(p_{\mathrm{B}}, p_{\mathrm{D}}, \lambda\right) \in \mathbf{Q}$, such that

$$
\begin{aligned}
\mathscr{A}(\overrightarrow{\boldsymbol{u}}, \overrightarrow{\boldsymbol{v}})+\mathscr{B}(\overrightarrow{\boldsymbol{v}}, \vec{p}) & =\mathscr{F}_{\phi}(\overrightarrow{\boldsymbol{v}}) & & \forall \overrightarrow{\boldsymbol{v}}:=\left(\boldsymbol{v}_{\mathrm{B}}, \boldsymbol{z}_{\mathrm{B}}, \boldsymbol{v}_{\mathrm{D}}\right) \in \mathbf{H}, \\
\mathscr{B}(\overrightarrow{\boldsymbol{u}}, \vec{q}) & =0 & & \forall \vec{q}:=\left(q_{\mathrm{B}}, q_{\mathrm{D}}, \lambda\right) \in \mathbf{Q},
\end{aligned}
$$

where the product spaces are

$$
\mathbf{H}:=\mathbf{H}_{\mathrm{B}}\left(\operatorname{div} ; \Omega_{\mathrm{B}}\right) \times \mathbf{H}_{0}\left(\operatorname{curl} ; \Omega_{\mathrm{B}}\right) \times \mathbf{H}_{\mathrm{D}}\left(\operatorname{div} ; \Omega_{\mathrm{D}}\right), \quad \mathbf{Q}:=\mathrm{L}^{2}\left(\Omega_{\mathrm{B}}\right) \times \mathrm{L}^{2}\left(\Omega_{\mathrm{D}}\right) \times \mathrm{H}^{1 / 2}(\Sigma),
$$

the bilinear forms $\mathscr{A}: \mathbf{H} \times \mathbf{H} \rightarrow \mathrm{R}$ and $\mathscr{B}: \mathbf{H} \times \mathbf{Q} \rightarrow \mathrm{R}$ are defined by

$$
\mathscr{A}(\overrightarrow{\boldsymbol{u}}, \overrightarrow{\boldsymbol{v}}):=\mu \int_{\Omega_{\mathrm{B}}} \mathbb{K}_{\mathrm{B}}^{-1} \boldsymbol{u}_{\mathrm{B}} \cdot \boldsymbol{v}_{\mathrm{B}}+\mu \int_{\Omega_{\mathrm{B}}} \boldsymbol{\omega}_{\mathrm{B}} \cdot z_{\mathrm{B}}+\mu \int_{\Omega_{\mathrm{B}}} \boldsymbol{v}_{\mathrm{B}} \cdot \operatorname{curl} \omega_{\mathrm{B}}
$$




$$
\begin{gathered}
-\mu \int_{\Omega_{\mathrm{B}}} \boldsymbol{u}_{\mathrm{B}} \cdot \operatorname{curl} z_{\mathrm{B}}+\mu \int_{\Omega_{\mathrm{D}}} \mathbb{K}_{\mathrm{D}}^{-1} \boldsymbol{u}_{\mathrm{D}} \cdot \boldsymbol{v}_{\mathrm{D}}, \\
\mathscr{B}(\overrightarrow{\boldsymbol{v}}, \vec{q}):=-\int_{\Omega_{\mathrm{B}}} q_{\mathrm{B}} \operatorname{div} \boldsymbol{v}_{\mathrm{B}}-\int_{\Omega_{\mathrm{D}}} q_{\mathrm{D}} \operatorname{div} \boldsymbol{v}_{\mathrm{D}}+\left\langle\boldsymbol{v}_{\mathrm{B}} \cdot \boldsymbol{n}-\boldsymbol{v}_{\mathrm{D}} \cdot \boldsymbol{n}, \boldsymbol{\xi}\right\rangle_{\Sigma},
\end{gathered}
$$

for all $\overrightarrow{\boldsymbol{v}} \in \mathbf{H}, \vec{q} \in \mathbf{Q}$, and $\mathscr{F} \in \mathbf{H}^{\prime}$ is the functional defined by

$$
\mathscr{F} \psi(\overrightarrow{\boldsymbol{v}}):=\int_{\Omega_{\mathrm{B}}} \psi f_{\mathrm{B}} \cdot \boldsymbol{v}_{\mathrm{B}}+\int_{\Omega_{\mathrm{D}}} \psi f_{\mathrm{D}} \cdot v_{\mathrm{D}} \quad \forall \overrightarrow{\boldsymbol{v}} \in \mathbf{H} .
$$

Next, we observe that the solution for 3.2 is not unique. Indeed, it suffices to consider $\vec{p}:=(c, c, c)$, with $c \in \mathrm{R}$ to see that $(\mathbf{0}, \vec{p})$ is also solution to the homogeneous system (see Alvarez et al. 2016b Theorem 3.1). In order to amend this, the formulation can be modified as follows. Find $(\overrightarrow{\boldsymbol{u}}, \vec{p}) \in \mathbf{H} \times \mathbf{Q}_{0}$, such that

$$
\begin{aligned}
\mathscr{A}(\overrightarrow{\boldsymbol{u}}, \overrightarrow{\boldsymbol{v}})+\mathscr{B}(\overrightarrow{\boldsymbol{v}}, \vec{p}) & =\mathscr{F}_{\phi}(\overrightarrow{\boldsymbol{v}}) & & \forall \overrightarrow{\boldsymbol{v}} \in \mathbf{H}, \\
\mathscr{B}(\overrightarrow{\boldsymbol{u}}, \vec{q}) & =0 & & \forall \vec{q} \in \mathbf{Q}_{0},
\end{aligned}
$$

where $\mathbf{Q}_{0}:=\mathrm{L}_{0}^{2}\left(\Omega_{\mathrm{B}}\right) \times \mathrm{L}^{2}\left(\Omega_{\mathrm{D}}\right) \times \mathrm{H}^{1 / 2}(\Sigma)$.

On the other hand, given $\boldsymbol{u}$ in a suitable space (to be made precise in Lemma 3.2 . below), testing with functions in $\mathrm{H}_{\Gamma_{0}}^{1}(\Omega)$, integrating by parts and using the boundary data, we deduce the following primal formulation for the transport problem: Find $\phi \in \mathrm{H}_{\Gamma_{0}}^{1}(\Omega)$ such that

$$
\mathscr{C}_{\boldsymbol{u}}(\phi, \psi)=\int_{\Omega} f_{\mathrm{bk}}(\phi) \boldsymbol{g} \cdot \nabla \psi \quad \forall \psi \in \mathrm{H}_{\Gamma_{0}}^{1}(\Omega),
$$

where, the form $\mathscr{C}_{\boldsymbol{u}}$ is defined by

$$
\mathscr{C}_{\boldsymbol{u}}(\phi, \psi):=\int_{\Omega} \vartheta(\phi) \nabla \phi \cdot \nabla \psi-\int_{\Omega} \phi \boldsymbol{u} \cdot \nabla \psi+\int_{\Omega} \beta \phi \psi \quad \forall \phi, \psi \in \mathrm{H}_{\Gamma_{0}}^{1}(\Omega)
$$

The mixed-primal formulation of our original coupled problem 2.1 - 2.3 ; $; 2.4$, reduces then to 3.4 - 3.5 , that is: Find $(\overrightarrow{\boldsymbol{u}}, \vec{p}, \phi) \in \mathbf{H} \times \mathbf{Q}_{0} \times \mathrm{H}_{\Gamma_{0}}^{1}(\Omega)$ such that

$$
\begin{aligned}
\mathscr{A}(\overrightarrow{\boldsymbol{u}}, \overrightarrow{\boldsymbol{v}})+\mathscr{B}(\overrightarrow{\boldsymbol{v}}, \vec{p}) & =\mathscr{F}_{\phi}(\overrightarrow{\boldsymbol{v}}) & \forall \overrightarrow{\boldsymbol{v}} \in \mathbf{H}, \\
\mathscr{B}(\overrightarrow{\boldsymbol{u}}, \vec{q}) & =0 & \forall \vec{q} \in \mathbf{Q}_{0}, \\
\mathscr{C}_{\boldsymbol{u}}(\phi, \psi) & =\int_{\Omega} f_{\mathrm{bk}}(\phi) \boldsymbol{g} \cdot \nabla \psi & \forall \psi \in \mathrm{H}_{\Gamma_{0}}^{1}(\Omega) .
\end{aligned}
$$

The well-posedness of 3.7 will be addressed in Sections 3.3 and 3.4 We anticipate now that the analysis of 3.5 (in particular, the estimation of the second term defining $\mathscr{C}_{\boldsymbol{u}}$ ) requires further regularity of the global velocity.

\subsection{Fixed-point strategy}

We describe a fixed-point framework for 3.7). We commence by introducing the operator $\mathbf{S}^{\text {flow }}: \mathrm{H}_{\Gamma_{0}}^{1}(\Omega) \longrightarrow$ $\mathbf{H}(\operatorname{div} ; \Omega)$ defined as

$$
\mathbf{S}^{\text {flow }}(\phi):=\boldsymbol{u}:=\left\{\begin{array}{lll}
\boldsymbol{u}_{\mathrm{B}} & \text { in } & \Omega_{\mathrm{B}} \\
\boldsymbol{u}_{\mathrm{D}} & \text { in } & \Omega_{\mathrm{D}}
\end{array} \quad \forall \phi \in \mathrm{H}_{\Gamma_{0}}^{1}(\Omega),\right.
$$

that gives the flow velocity, where the Brinkman and Darcy velocities $\boldsymbol{u}_{\mathrm{B}}, \boldsymbol{u}_{\mathrm{D}}$ are part of the unique solution, to be confirmed below, of the interfacial flow problem 3.4 for the given concentration $\phi$.

In turn, we also introduce the operator $\mathbf{S}^{\text {adv }}: \mathrm{H}_{\Gamma_{0}}^{1}(\Omega) \times \mathbf{H}(\operatorname{div} ; \Omega) \longrightarrow \mathrm{H}_{\Gamma_{0}}^{1}(\Omega)$ defined as

$$
\mathbf{S}^{\operatorname{adv}}(\phi, \boldsymbol{u}):=\widetilde{\phi} \quad \forall(\phi, \boldsymbol{u}) \in \mathrm{H}_{\Gamma_{0}}^{1}(\Omega) \times \mathbf{H}(\operatorname{div} ; \Omega),
$$

where, given $(\phi, u) \in \mathrm{H}_{\Gamma_{0}}^{1}(\Omega) \times \mathbf{H}(\operatorname{div} ; \Omega), \widetilde{\phi}$ is the unique solution (to be confirmed below) of the linear advection problem arising from $(3.5)$ after performing the following actions: replacing the nonlinear expression $\vartheta(\phi) \nabla \phi$ appearing in the first term of $\mathscr{C}_{\boldsymbol{u}}$ (cf. (3.6) by the linear one $\vartheta(\phi) \nabla \widetilde{\phi}$; incorporating $\boldsymbol{u}$ into the second term defining $\mathscr{C}_{\boldsymbol{u}}$ (cf. (3.6), thus making it bilinear; and realizing that the expression on the right-hand side 
of 3.5 becomes a linear functional of $\psi$. In this way, the aforementioned linear problem reduces to: Find $\widetilde{\phi} \in \mathrm{H}_{\Gamma_{0}}^{1}(\Omega)$ such that

$$
\mathscr{C}_{\boldsymbol{\phi}, \boldsymbol{u}}(\widetilde{\phi}, \widetilde{\psi})=\mathscr{H}_{\boldsymbol{\phi}}(\widetilde{\psi}) \quad \forall \widetilde{\psi} \in \mathrm{H}_{\Gamma_{0}}^{1}(\Omega),
$$

where, as previously described, the new bilinear form $\mathscr{C}_{\phi, \boldsymbol{u}}$ and linear functional $\mathscr{H}_{\phi}$ adopt the definitions

$$
\mathscr{C}_{\phi, \boldsymbol{u}}(\widetilde{\phi}, \widetilde{\psi}):=\int_{\Omega} \vartheta(\phi) \nabla \widetilde{\phi} \cdot \nabla \widetilde{\psi}-\int_{\Omega} \widetilde{\phi} \boldsymbol{u} \cdot \nabla \widetilde{\psi}+\int_{\Omega} \beta \widetilde{\phi} \widetilde{\psi} \quad \forall \widetilde{\phi}, \widetilde{\psi} \in \mathrm{H}_{\Gamma_{0}}^{1}(\Omega),
$$

and

$$
\mathscr{H}_{\phi}(\widetilde{\boldsymbol{\psi}}):=\int_{\Omega} f_{\mathrm{bk}}(\phi) \boldsymbol{g} \cdot \nabla \widetilde{\boldsymbol{\psi}} \quad \forall \widetilde{\boldsymbol{\psi}} \in \mathrm{H}_{\Gamma_{0}}^{1}(\Omega) .
$$

Here, we stress in advance that $\mathbf{S}^{\text {adv }}$ will be well-defined not in the whole space $\mathrm{H}_{\Gamma_{0}}^{1}(\Omega) \times \mathbf{H}(\operatorname{div} ; \Omega)$, but only in a subspace of it (see Lemma 3.2 below).

Finally, we define the operator $\mathbf{T}: \mathrm{H}_{\Gamma_{0}}^{1}(\Omega) \longrightarrow \mathrm{H}_{\Gamma_{0}}^{1}(\Omega)$ as

$$
\mathbf{T}(\phi):=\mathbf{S}^{\mathrm{adv}}\left(\phi, \mathbf{S}^{\text {flow }}(\phi)\right) \quad \forall \phi \in \mathrm{H}_{\Gamma_{0}}^{1}(\Omega),
$$

and realise that solving (3.7) is equivalent to finding $\phi \in \mathrm{H}_{\Gamma_{0}}^{1}(\Omega)$ such that

$$
\mathbf{T}(\phi)=\phi .
$$

Indeed, $\phi \in \mathrm{H}_{\Gamma_{0}}^{1}(\Omega)$ is solution of (3.12) if and only if $\phi=\mathbf{S}^{\mathrm{adv}}\left(\phi, \mathbf{S}^{\text {flow }}(\phi)\right)$, which means that, once $\boldsymbol{u}=$ $\mathbf{S}^{\text {flow }}(\phi)$ is obtained as part of the solution of 3.4 with the given $\phi$, and then employed jointly with $\phi$ to solve [3.8), the resulting solution of the latter is the same initial $\phi$. Equivalently, this says that

$$
\mathscr{C}_{\phi, \boldsymbol{u}}(\phi, \widetilde{\psi})=\mathscr{H}_{\phi}(\widetilde{\psi}) \quad \forall \widetilde{\psi} \in \mathrm{H}_{\Gamma_{0}}^{1}(\Omega),
$$

which, bearing in mind the definitions of $\mathscr{C}_{\boldsymbol{u}}$ (cf. 3.6) and $\mathscr{C}_{\phi, \boldsymbol{u}}$ (cf. 3.9), and redenoting $\widetilde{\psi}$ as simply $\psi$, can be rewritten as

$$
\mathscr{C}_{\boldsymbol{u}}(\phi, \psi)=\int_{\Omega} f_{\mathrm{bk}}(\phi) \boldsymbol{g} \cdot \nabla \psi \quad \forall \psi \in \mathrm{H}_{\Gamma_{0}}^{1}(\Omega),
$$

which confirms that $\phi$ is the solution of 3.5. Hence, $(\overrightarrow{\boldsymbol{u}}, \vec{p}, \phi) \in \mathbf{H} \times \mathbf{Q}_{0} \times \mathrm{H}_{\Gamma_{0}}^{1}(\Omega)$, with $(\overrightarrow{\boldsymbol{u}}, \vec{p})$ solving 3.4 for the given $\phi$, is solution of the original coupled problem (3.7).

\subsection{Well-posedness of the uncoupled problem}

In this section, we show that the uncoupled problems 3.4 and 3.8 are in fact well-posed. We begin the solvability analysis with the following result, whose proof is a direct consequence of (Alvarez et al. 2016b. Theorem 3.2). Let us remark that similar vorticity-based formulations for Brinkman-Darcy equations can be analysed using a different approach, as done recently in Anaya et al. (2019).

LEMma 3.1 For each $\phi \in \mathrm{H}_{\Gamma_{0}}^{1}(\Omega)$, problem 3.4 has a unique solution $(\overrightarrow{\boldsymbol{u}}, \vec{p}) \in \mathbf{H} \times \mathbf{Q}_{0}$. Moreover, there exists $C_{\mathrm{S}^{\text {flow }}}>0$, independent of $\phi$, such that

$$
\left\|\mathbf{S}^{\text {flow }}(\phi)\right\|_{\operatorname{div}, \Omega} \leqslant\|(\overrightarrow{\boldsymbol{u}}, \vec{p})\|_{\mathbf{H} \times \mathbf{Q}_{0}} \leqslant C_{\mathbf{S}^{\text {flow }}}\|\phi\|_{0, \Omega}\left\{\left\|\boldsymbol{f}_{\mathrm{B}}\right\|_{\infty, \Omega_{\mathrm{B}}}+\left\|\boldsymbol{f}_{\mathrm{D}}\right\|_{\infty, \Omega_{\mathrm{D}}}\right\}, \quad \forall \phi \in \mathrm{H}_{\Gamma_{0}}^{1}(\Omega) .
$$

For the purpose of the next result, which provides the solvability of the uncoupled problem [3.8, we require that the global velocity $\boldsymbol{u}$ belong to $\mathbf{H}(\operatorname{div} ; \Omega) \cap \mathbf{H}^{\delta}(\Omega)$ for some $\delta \in(0,1)$ (when $\left.n=2\right)$ or $\delta \in(1 / 2,1)$ (when $n=3$ ). In turn, according to the aforementioned range for $\delta$, we recall that the Sobolev embedding Theorem ( $c f$. Adams \& Fournier. 2003 Theorem 4.12, Quarteroni \& Valli, 1994, Theorem 1.3.4) establishes the continuous injection $\boldsymbol{i}_{\delta}: \mathbf{H}^{\delta}(\Omega) \longrightarrow \mathbf{L}^{\delta^{*}}(\Omega)$ with boundedness constant $\mathbf{C}_{\delta}^{*}$, where

$$
\delta^{*}:=\left\{\begin{array}{lll}
\frac{2}{1-\delta} & \text { if } & n=2 \\
\frac{6}{3-2 \delta} & \text { if } & n=3
\end{array}\right.
$$

and it also guarantees that the injection $\mathrm{i}: \mathrm{H}^{1}(\Omega) \rightarrow \mathrm{L}^{n / \delta}(\Omega)$ is compact, and hence continuous, with constant $\mathrm{C}_{\delta}$. In addition, we set $r_{0}:=\frac{\vartheta_{1}}{2 c_{p} \mathrm{C}_{\delta} \mathbf{C}_{\delta}^{*}}$, where $\vartheta_{1}$ and $c_{p}$ are the constants given in 2.5 and 2.8, respectively. 
LeMmA 3.2 Let $\phi \in \mathrm{H}_{\Gamma_{0}}^{1}(\Omega)$, and $\boldsymbol{u} \in \mathbf{H}(\operatorname{div} ; \Omega) \cap \mathbf{H}^{\delta}(\Omega)$ for some $\delta \in(0,1)$ (when $\left.n=2\right)$ or $\delta \in(1 / 2,1)$ (when $n=3$ ), such that $\|\boldsymbol{u}\|_{\delta, \Omega}<r_{0}$. Then, the problem 3.8 has a unique solution $\mathbf{S}^{\operatorname{adv}}(\phi, \boldsymbol{u}):=\widetilde{\phi} \in \mathrm{H}_{\Gamma_{0}}^{1}(\Omega)$. Moreover, there exists $C_{\mathbf{S} \text { adv }}>0$, independent of $(\phi, \boldsymbol{u})$, such that

$$
\left\|\mathbf{S}^{\mathrm{adv}}(\phi, \boldsymbol{u})\right\|_{1, \Omega} \leqslant C_{\mathbf{S}^{\mathrm{adv}}} \gamma_{2}|\Omega|^{1 / 2}|\boldsymbol{g}| .
$$

Proof. We first notice that $\mathscr{C}_{\boldsymbol{\phi}, \boldsymbol{u}}(c f$. [3.9) is clearly a bilinear form. In turn, employing the upper bound of $\vartheta$ (cf. 2.5), Cauchy-Schwarz's inequality, and Hölder's inequality, it readily follows from (3.9) that

$$
\left|\mathscr{C}_{\phi, \boldsymbol{u}}(\widetilde{\boldsymbol{\phi}}, \widetilde{\boldsymbol{\psi}})\right| \leqslant \vartheta_{2}|\widetilde{\boldsymbol{\phi}}|_{1, \Omega}|\widetilde{\boldsymbol{\psi}}|_{1, \Omega}+\|\widetilde{\boldsymbol{\phi}}\|_{\mathrm{L}^{2 q}(\Omega)}\|\boldsymbol{u}\|_{\mathbf{L}^{2 p}(\Omega)}|\widetilde{\boldsymbol{\psi}}|_{1, \Omega}+\beta\|\widetilde{\phi}\|_{0, \Omega}\|\widetilde{\psi}\|_{0, \Omega}
$$

where $p, q \in[1,+\infty)$ are such that $1 / p+1 / q=1$. Next, choosing $p$ such that $2 p=\delta^{*}(c f$. 3.14), it readily follows that

$$
2 q:=\frac{2 p}{p-1}=\frac{n}{\delta}
$$

The range of $\delta$ implies that we can apply the continuous injections $\boldsymbol{i}_{\delta}: \mathbf{H}^{\delta}(\Omega) \longrightarrow \mathbf{L}^{\delta^{*}}(\Omega)$, and i : $\mathrm{H}^{1}(\Omega) \rightarrow$ $\mathrm{L}^{n / \delta}(\Omega)$ in combination with the bound for $\|\boldsymbol{u}\|_{\delta, \Omega}$, to deduce from 3.16 the existence of a positive constant $\|\mathscr{C}\|$ (depending on $\vartheta_{1}, \vartheta_{2}, \beta,\left\|\boldsymbol{i}_{\delta}\right\|,\|\mathrm{i}\|$, and $c_{p}$ ) such that

$$
\left|\mathscr{C}_{\phi, \boldsymbol{u}}(\widetilde{\boldsymbol{\phi}}, \widetilde{\psi})\right| \leqslant\|\mathscr{C}\|\|\widetilde{\phi}\|_{1, \Omega}\|\widetilde{\psi}\|_{1, \Omega} \quad \forall \widetilde{\phi}, \widetilde{\psi} \in \mathrm{H}_{\Gamma_{0}}^{1}(\Omega) .
$$

This proves that $\mathscr{C}_{\phi, \boldsymbol{u}}$ is bounded independently of $\phi$ and $\boldsymbol{u}$. On the other hand, applying the same argument used for the derivation of second term on the right-hand side of (3.16), and using [3.14, , 3.17) and (2.8), we find that for each $\widetilde{\phi} \in \mathrm{H}_{\Gamma_{\mathrm{D}}}^{1}(\Omega)$ there holds

$$
\begin{aligned}
\mathscr{C}_{\phi, \boldsymbol{u}}(\widetilde{\boldsymbol{\phi}}, \widetilde{\phi}) & =\int_{\Omega} \vartheta(\phi)|\nabla \widetilde{\phi}|^{2}-\int_{\Omega} \widetilde{\phi} \boldsymbol{u} \cdot \nabla \widetilde{\phi}+\beta\|\widetilde{\phi}\|_{0, \Omega}^{2} \\
& \geqslant \vartheta_{1}|\widetilde{\phi}|_{1, \Omega}^{2}-\mathbf{C}_{\delta}^{*}\|\widetilde{\phi}\|_{\mathrm{L}^{n / \delta}(\Omega)}\|\boldsymbol{u}\|_{\delta, \Omega}|\widetilde{\phi}|_{1, \Omega}+\beta\|\widetilde{\phi}\|_{0, \Omega}^{2} \\
& \geqslant\left(\vartheta_{1}-c_{p} \mathrm{C}_{\delta} \mathbf{C}_{\delta}^{*}\|\boldsymbol{u}\|_{\delta, \Omega}\right)|\widetilde{\phi}|_{1, \Omega}^{2} \\
& \geqslant \frac{\vartheta_{1}}{2}|\widetilde{\phi}|_{1, \Omega}^{2} \geqslant \frac{\vartheta_{1}}{2 c_{p}^{2}}\|\widetilde{\phi}\|_{1, \Omega}^{2},
\end{aligned}
$$

which proves that $\mathscr{C}_{\boldsymbol{\phi}, \boldsymbol{u}}$ is $\mathrm{H}_{\Gamma_{0}}^{1}(\Omega)$-elliptic with constant $\widetilde{\alpha}:=\frac{\vartheta_{1}}{2 c_{p}^{2}}$, also independently of both $\phi$ and $\boldsymbol{u}$. Next, applying Cauchy-Schwarz inequality and the upper bound for $f_{\mathrm{bk}}$ given in 2.5 , we easily deduce that

$$
\left|\mathscr{H}_{\phi}(\widetilde{\psi})\right| \leqslant \gamma_{2}|\Omega|^{1 / 2}|\boldsymbol{g}|\|\widetilde{\psi}\|_{1, \Omega} \quad \forall \widetilde{\psi} \in \mathrm{H}_{\Gamma_{\mathrm{D}}}^{1}(\Omega),
$$

which says that $\mathscr{H}_{\phi} \in \mathrm{H}_{\Gamma_{0}}^{1}(\Omega)^{\prime}$ and $\left\|\mathscr{H}_{\phi}\right\| \leqslant \gamma_{2}|\Omega|^{1 / 2}|\boldsymbol{g}|$. Consequently, a direct application of the LaxMilgram Lemma implies the existence of a unique solution $\widetilde{\phi}:=\mathbf{S}^{\mathrm{adv}}(\phi, \boldsymbol{u}) \in \mathrm{H}_{\Gamma_{0}}^{1}(\Omega)$ of [3.8], and the corresponding continuous dependence result becomes 3.15 with $C_{\mathrm{S}^{\mathrm{adv}}}=\frac{1}{\widetilde{\alpha}}=\frac{2 c_{p}^{2}}{\vartheta_{1}}$.

REMARK 3.1 The bound for $\|\boldsymbol{u}\|_{\delta, \Omega}$ used in Lemma 3.2 could also have been taken as

$$
\|\boldsymbol{u}\|_{\delta, \Omega}<\varepsilon \frac{\vartheta_{1}}{c_{p} \mathrm{C}_{\delta} \mathbf{C}_{\delta}^{*}}
$$

with any $\varepsilon \in(0,1)$, however choosing simply $\varepsilon=\frac{1}{2}$ yields a joint maximisation of the ellipticity constant of $\mathscr{C}_{\phi, \boldsymbol{u}}$. In addition, dropping the term $\beta\|\widetilde{\phi}\|_{0, \Omega}^{2}$ in 3.18 we have assumed that $\beta$ is small and then utilised the Poincaré inequality (2.8). When $\beta$ is instead large, say $\beta \geqslant \vartheta_{1}$, then the aforementioned expression is maintained throughout the derivation of 3.18 , implying that the Poincaré inequality 2.8 is not required.

We end this section by introducing adequate regularity hypotheses on $\mathbf{S}^{\text {flow }}$ which will be employed to guarantee that the operator $\mathbf{T}$ is well-defined. In addition, we also assume sufficient regularity of $\mathbf{S}^{\text {adv }}$ in order to establish its Lipschitz continuity, and also that of $\mathbf{T}$. In fact, for the remainder of this paper we follow Alvarez et al. 2016a. Eq. (3.23) and Eq. (3.24)), and consider the following two hypotheses. 
REgUlaRity Hypothesis 3.1 For $f_{\mathrm{B}} \in \mathbf{L}^{\infty}\left(\Omega_{\mathrm{B}}\right), f_{\mathrm{D}} \in \mathbf{L}^{\infty}\left(\Omega_{\mathrm{D}}\right)$, and for each $\phi \in \mathrm{H}_{\Gamma_{0}}^{1}(\Omega)$ with $\|\phi\|_{1, \Omega} \leqslant r$, $r>0$ given, there holds $\mathbf{S}^{\text {flow }}(\phi) \in \mathbf{H}(\operatorname{div} ; \Omega) \cap \mathbf{H}^{\delta}(\Omega)$, for some $\delta \in(0,1)$ (when $\left.n=2\right)$ or $\delta \in(1 / 2,1)$ (when $n=3$ ), with

$$
\left\|\mathbf{S}^{\text {flow }}(\phi)\right\|_{\delta, \Omega} \leqslant \widehat{C}_{\mathbf{S}^{\text {flow }}}(r)\|\phi\|_{0, \Omega}\left\{\left\|\boldsymbol{f}_{\mathrm{B}}\right\|_{\infty, \Omega_{\mathrm{B}}}+\left\|\boldsymbol{f}_{\mathrm{D}}\right\|_{\infty, \Omega_{\mathrm{D}}}\right\},
$$

where $\widehat{C}_{\mathrm{S}^{\text {flow }}}(r)$ is a positive constant independent of $\phi$, but depending on the upper bound $r$ of its norm.

Regularity Hypothesis 3.2 For each $(\varphi, \boldsymbol{w}) \in \mathrm{H}_{\Gamma_{0}}^{1}(\Omega) \times\left(\mathbf{H}(\operatorname{div} ; \Omega) \cap \mathbf{H}^{\delta}(\Omega)\right)$, with $\delta \in(0,1)$ (when $n=2$ ) or $\delta \in(1 / 2,1)$ (when $n=3$ ), and $\|\varphi\|_{1, \Omega}+\|\boldsymbol{w}\|_{\operatorname{div}, \Omega}+\|\boldsymbol{w}\|_{\delta} \leqslant r, r>0$ given, there holds $\mathbf{S}^{\text {adv }}(\varphi, \boldsymbol{w}) \in$ $\mathrm{H}_{\Gamma_{0}}^{1+\delta}(\Omega)$, with

$$
\left\|\mathbf{S}^{\mathrm{adv}}(\varphi, \boldsymbol{w})\right\|_{1+\delta, \Omega} \leqslant \widehat{C}_{\mathbf{S}^{\mathrm{adv}}}(r) C(\Omega)|\boldsymbol{g}|
$$

where $C(\Omega)$ is a positive constant depending only on the domain $\Omega$, and $\widehat{C}_{\mathrm{S}^{\text {adv }}}(r)$ is a positive constant independent of $(\boldsymbol{\varphi}, \boldsymbol{w})$, but depending on the upper bound $r$ of its norm.

Hypothesis 3.1 will be needed in the proof of Lemma 3.3 More precisely, it is required to invoke Lemma 3.2. which is in turn crucial to prove that $\mathbf{T}$ is well-defined. Subsequently, the estimate (3.19) is employed in Lemma 3.5 to bound an expression of the form $\left\|\mathbf{S}^{\text {flow }}(\phi-\varphi)\right\|_{\mathbf{L}^{2 p}(\Omega)}$ in terms of $\left\|\mathbf{S}^{\text {flow }}(\phi-\varphi)\right\|_{\delta, \Omega}$, and hence depending on the right-hand side of 3.19 . In turn, the further regularity from Hypothesis 3.2 is used in the proof of Lemma 3.4 to bound an expression of the form $\left\|\nabla \mathbf{S}^{\mathrm{adv}}(\varphi, \boldsymbol{w})\right\|_{\mathbb{L}^{2 p}(\Omega)}$ in terms of $\left\|\mathbf{S}^{\mathrm{adv}}(\varphi, \boldsymbol{w})\right\|_{1+\delta, \Omega}$, which in the proof of Theorem 3.1 can be bounded by the data appearing in the right-hand side of 3.20 .

\subsection{Solvability of the fixed-point equation}

The well-posedness of the uncoupled problems (3.4) and 3.8) confirms that the operators $\mathbf{S}^{\text {flow }}, \mathbf{S}{ }^{\text {adv }}$ and $\mathbf{T}$ are well-defined. In order to address the solvability of the fixed-point equation $(3.12$, we verify the hypotheses of the Schauder fixed-point theorem (see, e.g. Ciarlet (2013) [Theorem 9.12-1(b)]).

We first define the following set, for any $r>0$

$$
\mathrm{W}^{r}:=\left\{\phi \in \mathrm{H}_{\Gamma_{0}}^{1}(\Omega): \quad\|\phi\|_{1, \Omega} \leqslant r\right\},
$$

and we have the following result.

LEMMA 3.3 Given $r>0$ and 3.21 , assume that

$$
\left\|\boldsymbol{f}_{\mathrm{B}}\right\|_{\infty, \Omega_{\mathrm{B}}}+\left\|\boldsymbol{f}_{\mathrm{D}}\right\|_{\infty, \Omega_{\mathrm{D}}}<\frac{r_{0}}{r \widehat{C}_{\mathbf{S}^{\text {flow }}}(r)} \quad \text { and } \quad C_{\mathrm{S}^{\text {adv }}} \gamma_{2}|\Omega|^{1 / 2}|\boldsymbol{g}| \leqslant r
$$

where $r_{0}$ is the constant specified right before Lemma 3.2 Then $\mathbf{T}\left(\mathrm{W}^{r}\right) \subseteq \mathrm{W}^{r}$.

Proof. Let us consider a given $\phi \in \mathrm{W}^{r}$. We then combine the arguments from Lemma 3.1, the estimate (3.19), the first condition in 3.22), and Lemma 3.2 to conclude that $\mathbf{S}^{\operatorname{adv}}\left(\phi, \mathbf{S}^{\text {flow }}(\phi)\right):=\mathbf{T}(\phi)$ is well-defined. Next, according to the definition of the operator $\mathbf{T}$ ( $c f$. 3.11), and the continuous dependence estimate 3.15, it readily follows that

$$
\|\mathbf{T}(\phi)\|_{1, \Omega}=\left\|\mathbf{S}^{\text {adv }}\left(\phi, \mathbf{S}^{\text {flow }}(\phi)\right)\right\|_{1, \Omega} \leqslant C_{\mathbf{S}^{\text {adv }}} \gamma_{2}|\Omega|^{1 / 2}|\boldsymbol{g}|,
$$

which, due to the second inequality in 3.22 , proves that $\mathbf{T}(\phi) \in \mathrm{W}^{r}$, thus finishing the proof.

Our next goal is to establish the continuity and compactness of $\mathbf{T}$, which is addressed in the following two lemmas.

Lemma 3.4 There exists a positive constant $\widetilde{C}>0$, depending on $L_{f_{\mathrm{bk}}}, L_{\vartheta}, \mathbf{C}_{\delta}^{*}, \widetilde{\alpha}(c f$. 2.7), 2.6, Lemma 3.2 , and the boundedness constant $\mathrm{C}_{\delta}^{*}$ of the injection $\mathrm{i}_{\delta}: \mathrm{H}^{\delta}(\Omega) \rightarrow \mathrm{L}^{\delta^{*}}(\Omega)$, such that for all $(\phi, u),(\varphi, \boldsymbol{w}) \in$ $\mathrm{H}_{\Gamma_{0}}^{1}(\Omega) \times\left(\mathbf{H}(\operatorname{div} ; \Omega) \cap \mathbf{H}^{\delta}(\Omega)\right)$, with $\|\boldsymbol{u}\|_{\delta, \Omega},\|\boldsymbol{w}\|_{\delta, \Omega}<r_{0}(c f$. Lemma 3.2 , there holds

$$
\begin{gathered}
\left\|\mathbf{S}^{\operatorname{adv}}(\phi, \boldsymbol{u})-\mathbf{S}^{\operatorname{adv}}(\varphi, \boldsymbol{w})\right\|_{1, \Omega} \leqslant \widetilde{C}\left\{|\boldsymbol{g}|\|\boldsymbol{\phi}-\varphi\|_{0, \Omega}+\left\|\mathbf{S}^{\operatorname{adv}}(\boldsymbol{\varphi}, \boldsymbol{w})\right\|_{\mathrm{L}^{n / \delta}(\Omega)}\|\boldsymbol{u}-\boldsymbol{w}\|_{\delta, \Omega}\right. \\
\left.+\left\|\mathbf{S}^{\operatorname{adv}}(\boldsymbol{\varphi}, \boldsymbol{w})\right\|_{1+\delta, \Omega}\|\phi-\varphi\|_{\mathrm{L}^{n / \delta}(\Omega)}\right\} .
\end{gathered}
$$

Proof. Given $(\phi, \boldsymbol{u}),(\boldsymbol{\varphi}, \boldsymbol{w})$ as stated, we let $\widetilde{\phi}:=\mathbf{S}^{\mathrm{adv}}(\boldsymbol{\phi}, \boldsymbol{u})$ and $\widetilde{\varphi}:=\mathbf{S}^{\mathrm{adv}}(\varphi, \boldsymbol{w})$, that is $(c f$. 3.8)

$$
\mathscr{C}_{\phi, u}(\widetilde{\phi}, \widetilde{\psi})=\mathscr{H}_{\phi}(\widetilde{\psi}) \quad \text { and } \quad \mathscr{C}_{\varphi, w}(\widetilde{\varphi}, \widetilde{\psi})=\mathscr{H}_{\varphi}(\widetilde{\psi}) \quad \forall \widetilde{\psi} \in \mathrm{H}_{\Gamma_{0}}^{1}(\Omega)
$$


According to the ellipticity of $\mathscr{C}_{\boldsymbol{\phi}, \boldsymbol{u}}$ with constant $\widetilde{\alpha}$, we can subtract and add $\mathscr{H}_{\varphi}(\widetilde{\phi}-\widetilde{\varphi})=\mathscr{C}_{\varphi, w}(\widetilde{\varphi}, \widetilde{\phi}-\widetilde{\varphi})$ to the previous expression, leading to

$$
\begin{aligned}
\widetilde{\alpha}\|\widetilde{\phi}-\widetilde{\varphi}\|_{1, \Omega}^{2} \leqslant & \mathscr{C}_{\phi, u}(\widetilde{\phi}, \widetilde{\phi}-\widetilde{\varphi})-\mathscr{C}_{\phi, u}(\widetilde{\varphi}, \widetilde{\phi}-\widetilde{\varphi}) \\
= & \mathscr{H}_{\phi}(\widetilde{\phi}-\widetilde{\varphi})-\mathscr{H}_{\varphi}(\widetilde{\phi}-\widetilde{\varphi})+\mathscr{C}_{\varphi, \boldsymbol{w}}(\widetilde{\varphi}, \widetilde{\phi}-\widetilde{\varphi})-\mathscr{C}_{\phi, \boldsymbol{u}}(\widetilde{\varphi}, \widetilde{\phi}-\widetilde{\varphi}) \\
= & \int_{\Omega}\left(f_{\mathrm{bk}}(\phi)-f_{\mathrm{bk}}(\varphi)\right) \boldsymbol{g} \cdot \nabla(\widetilde{\phi}-\widetilde{\varphi})+\int_{\Omega} \widetilde{\varphi}(\boldsymbol{u}-\boldsymbol{w}) \cdot \nabla(\widetilde{\phi}-\widetilde{\varphi}) \\
& +\int_{\Omega}(\vartheta(\varphi)-\vartheta(\phi)) \nabla \widetilde{\varphi} \cdot \nabla(\widetilde{\phi}-\widetilde{\varphi}),
\end{aligned}
$$

where for the last equality we have employed definitions 3.9 and 3.10. Then we can apply Cauchy-Schwarz's inequality, Hölder's inequality, Hypothesis 3.2 the Lipschitz-continuity $(2.6)-(2.7)$, which allows us to treat the last two terms in 3.24, much as in 3.18). Eventually, these steps imply the estimate

$$
\begin{aligned}
\widetilde{\alpha}\|\widetilde{\phi}-\widetilde{\varphi}\|_{1, \Omega}^{2} \leqslant & \left\{L_{f_{\mathrm{bk}}}|\boldsymbol{g}|\|\phi-\varphi\|_{0, \Omega}+\mathbf{C}_{\delta}^{*}\|\widetilde{\varphi}\|_{\mathrm{L}^{n / \delta}(\Omega)}\|\boldsymbol{u}-\boldsymbol{w}\|_{\delta, \Omega}\right\}|\widetilde{\boldsymbol{\phi}}-\widetilde{\varphi}|_{1, \Omega} \\
& +L_{\vartheta} \mathrm{C}_{\delta}^{*}\|\phi-\varphi\|_{\mathrm{L}^{n / \delta}(\Omega)}\|\nabla \widetilde{\varphi}\|_{\delta, \Omega}|\widetilde{\boldsymbol{\phi}}-\widetilde{\varphi}|_{1, \Omega},
\end{aligned}
$$

and combining inequalities (3.24) and 3.25 readily gives 3.23 , thus finishing the proof.

The following result is a straightforward consequence of Lemma 3.4

LEMMA 3.5 Given $r>0$ and the definition of $\mathrm{W}^{r}$ from 3.21 , let us assume the condition 3.22 . Then, for all $\phi, \varphi \in \mathrm{H}_{\Gamma_{0}}^{1}(\Omega)$, there holds

$$
\begin{aligned}
\|\mathbf{T}(\phi)-\mathbf{T}(\varphi)\|_{1, \Omega} \leqslant & \left\{\widetilde{C}|\boldsymbol{g}|+r \widetilde{C} \widehat{C}_{\mathbf{S}^{\text {flow }}}(r)\left(\left\|\boldsymbol{f}_{\mathrm{B}}\right\|_{\infty, \Omega_{\mathrm{B}}}+\left\|\boldsymbol{f}_{\mathrm{D}}\right\|_{\infty, \Omega_{\mathrm{D}}}\right)\|\mathbf{T}(\varphi)\|_{\mathrm{L}^{n / \delta}(\Omega)}\right\}\|\phi-\varphi\|_{0, \Omega} \\
& +\widetilde{C}\|\mathbf{T}(\varphi)\|_{1+\delta, \Omega}\|\phi-\varphi\|_{\mathrm{L}^{n / \delta}(\Omega)},
\end{aligned}
$$

where $\widetilde{C}$ and $\widehat{C}_{\mathbf{S}^{\text {flow }}}(r)$ are the constants given in Lemma 3.4 and estimate 3.19 , respectively.

Proof. It suffices to recall from Section 3.2 that $\mathbf{T}(\phi)=\mathbf{S}^{\mathrm{adv}}\left(\phi, \mathbf{S}^{\text {flow }}(\phi)\right) \quad \forall \phi \in \mathrm{H}_{\Gamma_{0}}^{1}(\Omega)$, and then directly apply Lemmas 3.3 .3 .4 the linearity of $\mathbf{S}^{\text {thow }}$, and the estimate 3.19 .

The announced properties of $\mathbf{T}$ are proved now.

LEMMA 3.6 The map $\mathbf{T}: \mathrm{W}^{r} \rightarrow \mathrm{W}^{r}$ is continuous and $\overline{\mathbf{T}\left(\mathrm{W}^{r}\right)}$ is compact.

Proof. It follows almost verbatim as in (Alvarez et al. 2015. Lemma 3.12). Essentially, the proof is a consequence of the Rellich-Kondrachov compactness Theorem (Adams \& Fournier. 2003. Theorem 6.3), the specified range of the constant $\delta$ involved in Hypotheses 3.1 and 3.2 and the well-known fact that every bounded sequence in a Hilbert space has a weakly convergent subsequence.

Finally, the main result of this section is stated as follows.

THEOREM 3.1 Assume that the hypotheses of the Lemmas 3.3 3.6 are met. Then, the mixed-primal problem 3.7) has at least one solution $(\overrightarrow{\boldsymbol{u}}, \vec{p}, \phi) \in \mathbf{H} \times \mathbf{Q}_{0} \times \mathrm{H}_{\Gamma_{0}}^{1}(\Omega)$ with $\phi \in \mathrm{W}^{r}$, satisfying the continuous dependences

$$
\begin{gathered}
\|(\overrightarrow{\boldsymbol{u}}, \vec{p})\|_{\mathbf{H} \times \mathbf{Q}_{0}} \leqslant r C_{\mathbf{S}^{\text {flow }}}\left\{\left\|\boldsymbol{f}_{\mathrm{B}}\right\|_{\infty, \Omega_{\mathrm{B}}}+\left\|\boldsymbol{f}_{\mathrm{D}}\right\|_{\infty, \Omega_{\mathrm{D}}}\right\}, \\
\|\phi\|_{1, \Omega} \leqslant C_{\mathbf{S}^{\mathrm{adv}}} \gamma_{2}|\Omega|^{1 / 2}|\boldsymbol{g}|,
\end{gathered}
$$

where $C_{\mathbf{S}^{\text {flow }}}$ and $C_{\mathbf{S}^{\text {adv }}}$ are the constants specified in Lemmas 3.1 and 3.2 respectively. Moreover, if the data $\boldsymbol{f}_{\mathrm{B}}, \boldsymbol{f}_{\mathrm{D}}$ and $\boldsymbol{g}$ are sufficiently small so that

$$
\widetilde{C}\left(1+\widehat{C}_{\mathbf{S}^{\text {adv }}}(r) \mathrm{C}_{\delta} C(\Omega)\right)|\boldsymbol{g}|+r^{2} \widetilde{C} \mathrm{C}_{\delta} \widehat{C}_{\mathbf{S}^{\text {flow }}}(r)\left(\left\|\boldsymbol{f}_{\mathrm{B}}\right\|_{\infty, \Omega_{\mathrm{B}}}+\left\|\boldsymbol{f}_{\mathrm{D}}\right\|_{\infty, \Omega_{\mathrm{D}}}\right)<1,
$$

where $\widetilde{C}, \widehat{C}_{\mathbf{S}^{\text {flow }}}(r), \widehat{C}_{\mathrm{S}^{\text {adv }}}(r)$ and $C(\Omega)$ are the constants from Lemma 3.4 and estimates 3.19) and 3.20), respectively, and $\mathrm{C}_{\delta}$ is the boundedness constant of the continuous injection of $\mathrm{H}^{1}(\Omega)$ into $\mathrm{L}^{n / \delta}(\Omega)$; then the solution $\phi$ is unique in $\mathrm{W}^{r}$.

Proof. According to the equivalence between (3.7) and the fixed-point equation (3.12), and thanks to Lemmas 3.3 and 3.6 the existence of solution is a direct consequence of the Schauder fixed-point theorem (Ciarlet. 
2013. Theorem 9.12-1(b)). In turn, the estimates 3.27) and 3.28 follow from 3.13 and 3.15, respectively. Finally, given another solution $\varphi \in \mathrm{W}^{r}$ of 3.12), the estimates 3.26 , together with the bounds

$$
\|\mathbf{T}(\varphi)\|_{1, \Omega}=\|\varphi\|_{1, \Omega} \leqslant r, \quad\|\varphi\|_{1+\delta, \Omega} \leqslant \widehat{C}_{\mathbf{S}^{\text {adv }}}(r) C(\Omega)|\boldsymbol{g}| \quad(c f .3 .20),
$$

and

$$
\|\varphi\|_{\mathrm{L}^{n / \delta}(\Omega)} \leqslant \mathrm{C}_{\delta}\|\varphi\|_{1, \Omega} \quad \forall \varphi \in \mathrm{H}^{1}(\Omega),
$$

confirm 3.29 as a sufficient condition for uniqueness.

Alternatively to the fixed-point analysis developed in this section, one could appeal to Banach's fixedpoint theory starting from the same setting, that is, exactly the same fixed-point equation of Section 3.2 It is also possible to define a different Picard problem where the global velocity is the fixed-point, or a completely different structure by appealing to monolithic approaches that do not separate flow from transport equations. Another option is to perform a linearisation such that also the linear parts of the global flux of the nonlinear advection equation are now part of the bilinear form. That is, moving terms from (3.10) to (3.9). These (or many other possible) choices can be explored case by case, especially in terms of the structure of the solvability analysis, but also regarding the suitability in light of the specific application. For instance, the strength of the coupling mechanisms can vary substantially from one flow regime to another. The strategy we have adopted here is adequate at least for the regime of laminar flow and transport dominated by nonlinear diffusion.

\section{Galerkin scheme}

Let $\mathscr{T}_{h}$ be a regular family of triangulations of $\bar{\Omega}_{\mathrm{B}} \cup \bar{\Omega}_{\mathrm{D}}$ by tetrahedra $K$ of diameter $h_{K}$ with meshsize $h:=$ $\max \left\{h_{K}: K \in \mathscr{T}_{h}\right\}$, such that $\mathscr{T}_{h}\left(\Omega_{\star}\right):=\left\{K \in \mathscr{T}_{h}: K \subseteq \bar{\Omega}_{\star}\right\}$ is a triangulation of $\Omega_{\star}$ for each $\star \in\{\mathrm{B}, \mathrm{D}\}$. We further denote by $\mathscr{T}_{h}(\Sigma)$ the triangulation on $\Sigma$ induced by $\mathscr{T}_{h}$ (either from $\Omega_{\mathrm{B}}$ or $\Omega_{\mathrm{D}}$ ). Also, we introduce an independent partition $\mathscr{T}_{\tilde{h}}(\Sigma)$ of $\Sigma$ by triangles $\widetilde{T}$ of diameter $h_{\widetilde{T}}$, and define $\tilde{h}:=\max \left\{h_{\widetilde{T}}: \widetilde{T} \in \mathscr{T}_{\tilde{h}}(\Sigma)\right\}$.

Let us now consider finite dimensional subspaces of the test and trial spaces appearing in Section 3 .

$$
\begin{aligned}
\mathbf{H}_{h}^{\mathrm{B}} \subseteq \mathbf{H}_{\mathrm{B}}\left(\operatorname{div} ; \Omega_{\mathrm{B}}\right), \quad \mathbf{H}_{0, h}^{\mathrm{B}} \subseteq \mathbf{H}_{0}\left(\mathbf{c u r l} ; \Omega_{\mathrm{B}}\right), \quad \mathbf{H}_{h}^{\mathrm{D}} \subseteq \mathbf{H}_{\mathrm{D}}\left(\operatorname{div} ; \Omega_{\mathrm{D}}\right), \quad \mathbf{X}_{h} \subset \mathbf{H}(\operatorname{div} ; \Omega), \\
\mathrm{Q}_{h}^{\mathrm{B}} \subseteq \mathrm{L}^{2}\left(\Omega_{\mathrm{B}}\right), \quad \mathrm{Q}_{h}^{\mathrm{D}} \subseteq \mathrm{L}^{2}\left(\Omega_{\mathrm{D}}\right), \quad \mathrm{Q}_{h}^{\Sigma} \subseteq \mathrm{H}^{1 / 2}(\Sigma), \quad \mathrm{H}_{h}^{\phi} \subseteq \mathrm{H}_{\Gamma_{0}}^{1}(\Omega) .
\end{aligned}
$$

Hence, setting the global spaces

$$
\mathbf{H}_{h}:=\mathbf{H}_{h}^{\mathrm{B}} \times \mathbf{H}_{0, h}^{\mathrm{B}} \times \mathbf{H}_{h}^{\mathrm{D}} \quad \text { and } \quad \mathbf{Q}_{0, h}:=\mathrm{Q}_{h, 0}^{\mathrm{B}} \times \mathrm{Q}_{h}^{\mathrm{D}} \times \mathrm{Q}_{h}^{\Sigma},
$$

the Galerkin scheme for 3.7) becomes: Find $\left(\overrightarrow{\boldsymbol{u}}_{h}, \vec{p}_{h}, \phi_{h}\right) \in \mathbf{H}_{h} \times \mathbf{Q}_{0, h} \times \mathrm{H}_{h}^{\phi}$ such that

$$
\begin{aligned}
\mathscr{A}\left(\overrightarrow{\boldsymbol{u}}_{h}, \overrightarrow{\boldsymbol{v}}_{h}\right)+\mathscr{B}\left(\overrightarrow{\boldsymbol{v}}_{h}, \vec{p}_{h}\right) & =\mathscr{F}_{\phi_{h}}\left(\overrightarrow{\boldsymbol{v}}_{h}\right) & & \forall \overrightarrow{\boldsymbol{v}}_{h} \in \mathbf{H}_{h}, \\
\mathscr{B}\left(\overrightarrow{\boldsymbol{u}}_{h}, \vec{q}_{h}\right) & =0 & & \forall \vec{q}_{h} \in \mathbf{Q}_{0, h}, \\
\mathscr{C}_{\boldsymbol{u}_{h}}\left(\phi_{h}, \psi_{h}\right) & =\mathscr{H}_{\phi_{h}}\left(\psi_{h}\right) & & \forall \psi_{h} \in \mathrm{H}_{h}^{\phi} .
\end{aligned}
$$

In order to guarantee the well-posedness of the Galerkin scheme 4.2, the subspaces introduced in 4.1 can be chosen as follows (see (Alvarez et al. 2016b. Section 4.1))

$$
\begin{aligned}
\mathbf{H}_{h}^{\star} & :=\left\{\boldsymbol{v}_{h}^{\star} \in \mathbf{H}_{\star}\left(\operatorname{div} ; \Omega_{\star}\right):\left.\quad \boldsymbol{v}_{h}^{\star}\right|_{K} \in \mathbb{R T}_{0}(K) \quad \forall K \in \mathscr{T}_{h}\left(\Omega_{\star}\right)\right\}, \\
\mathrm{Q}_{h}^{\star} & :=\left\{q_{h} \in \mathrm{L}^{2}\left(\Omega_{\star}\right):\left.\quad q_{h}\right|_{K} \in P_{0}(K) \quad \forall K \in \mathscr{T}_{h}\left(\Omega_{\star}\right)\right\}, \\
\mathrm{Q}_{h, 0}^{\star} & :=\mathrm{Q}_{h}^{\star} \cap \mathrm{L}_{0}^{2}\left(\Omega_{\star}\right), \\
\mathrm{H}_{h}^{\phi} & :=\left\{\psi_{h} \in C(\Omega) \cap \mathrm{H}_{\Gamma_{0}}^{1}(\Omega):\left.\quad \psi_{h}\right|_{K} \in \mathrm{P}_{1}(K) \quad \forall K \in \mathscr{T}_{h}\right\},
\end{aligned}
$$

with $\star \in\{\mathrm{B}, \mathrm{D}\}$, and where for any $K \in \mathscr{T}_{h}\left(\Omega_{\star}\right)$, we denote

$$
\mathbb{R T}_{0}(K):=\mathbf{P}_{0}(K) \oplus P_{0}(K) \boldsymbol{x},
$$

as the local Raviart-Thomas space of lowest order. In addition, we set

$$
\mathbf{H}_{0, h}^{\mathrm{B}}:=\left\{z_{h}^{\mathrm{B}} \in \mathbf{H}_{0}\left(\text { curl; } \Omega_{\mathrm{B}}\right):\left.\quad z_{h}^{\mathrm{B}}\right|_{K} \in \mathbb{N D}_{1}(K) \quad \forall K \in \mathscr{T}_{h}\left(\Omega_{\mathrm{B}}\right)\right\},
$$


where for any $K \in \mathscr{T}_{h}\left(\Omega_{\mathrm{B}}\right)$, the local edge space of Nédélec type is

$$
\mathbb{N D}_{1}(K):=\mathbf{P}_{0}(K) \oplus \mathbf{P}_{0}(K) \times \boldsymbol{x}=\left\{w: K \rightarrow \mathbb{C}^{3}: \quad w(\boldsymbol{x})=\boldsymbol{a}+\boldsymbol{b} \times \boldsymbol{x} \quad \forall \boldsymbol{x} \in K, \boldsymbol{a}, \boldsymbol{b} \in \mathbb{C}^{3}\right\} .
$$

In turn, we set $\mathbf{X}_{h}:=\mathbf{1}_{\Omega_{\mathrm{B}}} \mathbf{H}_{h}^{\mathrm{B}}+\mathbf{1}_{\Omega_{\mathrm{D}}} \mathbf{H}_{h}^{\mathrm{D}}$, whereas for the interfacial pressure, we consider the finite element space

$$
\mathrm{Q}_{\tilde{h}}^{\Sigma}:=\left\{\lambda_{\tilde{h}} \in \mathscr{C}^{0}(\Sigma):\left.\quad \lambda_{\tilde{h}}\right|_{\tilde{T}} \in P_{1}(\tilde{T}) \quad \forall \tilde{T} \in \mathscr{T}_{\tilde{h}}(\Sigma)\right\}
$$

\subsection{Discrete fixed-point strategy}

A first important remark is that the hypotheses of additional regularity that we employed to prove Lemmas 3.2 and 3.4 are neither needed nor applicable in the discrete case. In consequence, it is not possible to reuse the same fixed-point strategy from Section 3.2 Instead, we require that the operator associated with the discrete version of problem 3.4 is uniformly bounded. For simplicity of the presentation, the fixed-point scheme and its analysis will be restricted to the $2 \mathrm{D}$ case. Given $r>0$, we proceed to define

$$
\mathbf{Z}_{h}^{r}:=\left\{\boldsymbol{u}_{h} \in \mathbf{X}_{h}: \quad\left\|\boldsymbol{u}_{h}\right\|_{\star} \leqslant r\right\}
$$

where $\|\cdot\|_{\star}:=\|\cdot\|_{\operatorname{div}, \Omega}+\|\cdot\|_{\mathbf{L}^{s}(\Omega)}$, with $s>2$. We anticipate that the stipulated range for $s$ will allow us to invoke certain Sobolev embeddings in the subsequent analysis, more precisely, in the proof of Lemma 4.2 and Theorem 4.1, below.

We now set $Y_{h}:=\mathrm{H}_{h}^{\phi} \times \mathbf{Z}_{h}^{r}$ and introduce the operator $\mathbf{S}_{h}^{\mathrm{adv}}: Y_{h} \rightarrow \mathrm{H}_{h}^{\phi}$ defined by

$$
\mathbf{S}_{h}^{\mathrm{adv}}\left(\phi_{h}, \boldsymbol{u}_{h}\right):=\varphi_{h} \quad \forall\left(\phi_{h}, \boldsymbol{u}_{h}\right) \in Y_{h},
$$

where $\varphi_{h}$ is the unique solution (to be established below) of the linear problem: Find $\varphi_{h} \in \mathrm{H}_{h}^{\phi}$ such that

$$
\mathscr{C}_{\phi_{h}, u_{h}}\left(\varphi_{h}, \psi_{h}\right)=\mathscr{H}_{\phi_{h}}\left(\psi_{h}\right) \quad \forall \psi_{h} \in \mathrm{H}_{h}^{\phi},
$$

for given $\left(\phi_{h}, \boldsymbol{u}_{h}\right)$, where

$$
\mathscr{C}_{\phi_{h}, \boldsymbol{u}_{h}}\left(\varphi_{h}, \psi_{h}\right):=\int_{\Omega} \vartheta\left(\phi_{h}\right) \nabla \varphi_{h} \cdot \nabla \psi_{h}-\int_{\Omega} \varphi_{h} u_{h} \cdot \nabla \psi_{h}+\int_{\Omega} \beta \varphi_{h} \psi_{h} \quad \forall \varphi_{h}, \psi_{h} \in \mathrm{H}_{h}^{\phi},
$$

and

$$
\mathscr{H}_{\phi_{h}}\left(\psi_{h}\right):=\int_{\Omega} f_{\mathrm{bk}}\left(\phi_{h}\right) \boldsymbol{g} \cdot \nabla \psi_{h} \quad \forall \psi_{h} \in \mathrm{H}_{h}^{\phi} .
$$

In turn, we define the operator $\mathbf{S}_{h}^{\text {flow }}: \mathrm{H}_{h}^{\phi} \rightarrow \mathbf{X}_{h}$ as

$$
\mathbf{S}_{h}^{\text {flow }}\left(\varphi_{h}\right):=\boldsymbol{w}_{h} \quad \forall \varphi_{h} \in \mathrm{H}_{h}^{\phi},
$$

where $\left.\boldsymbol{w}_{h}\right|_{\Omega_{\mathrm{B}}}=\boldsymbol{w}_{h}^{\mathrm{B}}$ and $\left.\boldsymbol{w}_{h}\right|_{\Omega_{\mathrm{D}}}=\boldsymbol{w}_{h}^{\mathrm{D}}$ are the first and third components of $\overrightarrow{\boldsymbol{w}}_{h} \in \mathbf{H}_{h}$, which in turn is the first component of the unique solution (to be confirmed below) of the discrete problem associated with (3.4): Find $\left(\overrightarrow{\boldsymbol{w}}_{h}, \vec{r}_{h}\right) \in \mathbf{H}_{h} \times \mathbf{Q}_{0, h}$, such that

$$
\begin{array}{rlrl}
\mathscr{A}\left(\overrightarrow{\boldsymbol{w}}_{h}, \overrightarrow{\boldsymbol{v}}_{h}\right)+\mathscr{B}\left(\overrightarrow{\boldsymbol{v}}_{h}, \vec{r}_{h}\right) & =\mathscr{F} \varphi_{h}\left(\overrightarrow{\boldsymbol{v}}_{h}\right) & \forall \overrightarrow{\boldsymbol{v}}_{h} \in \mathbf{H}_{h}, \\
\mathscr{B}\left(\overrightarrow{\boldsymbol{w}}_{h}, \vec{q}_{h}\right)=0 & \forall \vec{q}_{h} \in \mathbf{Q}_{0, h},
\end{array}
$$

with $\varphi_{h}$ given. Therefore, by introducing the operator $\mathbf{T}_{h}: Y_{h} \rightarrow \mathrm{H}_{h}^{\phi} \times \mathbf{X}_{h}$ as

$$
\mathbf{T}_{h}\left(\phi_{h}, \boldsymbol{u}_{h}\right):=\left(\mathbf{S}_{h}^{\mathrm{adv}}\left(\phi_{h}, \boldsymbol{u}_{h}\right), \mathbf{S}_{h}^{\mathrm{flow}}\left(\mathbf{S}_{h}^{\mathrm{adv}}\left(\phi_{h}, \boldsymbol{u}_{h}\right)\right)\right) \quad \forall\left(\phi_{h}, \boldsymbol{u}_{h}\right) \in Y_{h},
$$

we see that solving (4.2) is equivalent to finding a fixed point of $\mathbf{T}_{h}$, that is: Find $\left(\phi_{h}, \boldsymbol{u}_{h}\right) \in Y_{h}$ such that

$$
\mathbf{T}_{h}\left(\phi_{h}, \boldsymbol{u}_{h}\right)=\left(\phi_{h}, \boldsymbol{u}_{h}\right)
$$

Certainly, all the above makes sense if we guarantee that the discrete problems 4.4) and 4.6 are well-posed. This is precisely the purpose of the next section. 


\subsection{Well-posedness of the uncoupled problem}

In this section, we establish the well-posedness of both 4.6 and 4.4, thus confirming that $\mathbf{S}_{h}^{\text {flow }}, \mathbf{S}_{h}^{\text {adv }}$, and hence $\mathbf{T}_{h}$, are well-defined.

LEMMA 4.1 For each $\phi_{h} \in \mathrm{H}_{h}^{\phi}$, the problem 4.6 has a unique solution $\left(\overrightarrow{\boldsymbol{u}}_{h}, \vec{p}_{h}\right) \in \mathbf{H}_{h} \times \mathbf{Q}_{0, h}$. Moreover, there exists a positive constant $C_{2}$, independent of $h$, such that

$$
\left\|\mathbf{S}_{h}^{\text {flow }}\left(\phi_{h}\right)\right\|_{\text {div }, \Omega} \leqslant\left\|\left(\overrightarrow{\boldsymbol{u}}_{h}, \vec{p}_{h}\right)\right\|_{\mathbf{H} \times \mathbf{Q}_{0}} \leqslant C_{2}\left\|\phi_{h}\right\|_{0, \Omega}\left\{\left\|\boldsymbol{f}_{\mathrm{B}}\right\|_{\infty, \Omega_{\mathrm{B}}}+\left\|\boldsymbol{f}_{\mathrm{D}}\right\|_{\infty, \Omega_{\mathrm{D}}}\right\} \quad \forall \phi_{h} \in \mathrm{H}_{h}^{\phi} .
$$

Proof. It follows directly from (Alvarez et al. 2016b Theorem 4.1).

LEMMA 4.2 Assume that $r \in\left(0, \frac{\vartheta_{1}}{2 \mathrm{C}_{s} c_{p}}\right)$, where $\mathrm{C}_{s}$ is the boundedness constant of the injection $\mathrm{i}_{s}: \mathrm{H}^{1}(\Omega) \rightarrow$ $\mathrm{L}^{\frac{2 s}{s-2}}(\Omega)$, with $s>2$. Then, for each $\left(\phi_{h}, \boldsymbol{u}_{h}\right) \in Y_{h}$, we see that problem (4.4) has a unique solution $\mathbf{S}_{h}^{\mathrm{adv}}\left(\phi_{h}, \boldsymbol{u}_{h}\right):=$ $\varphi_{h} \in \mathrm{H}_{h}^{\phi}$. Moreover, by denoting $C_{1}:=\frac{1}{\widetilde{\alpha}}$, with $\widetilde{\alpha}$ as in the proof of Lemma 3.2 there holds

$$
\left\|\mathbf{S}_{h}^{\operatorname{adv}}\left(\phi_{h}, \boldsymbol{u}_{h}\right)\right\|_{1, \Omega} \leqslant C_{1} \gamma_{2}|\Omega|^{1 / 2}|\boldsymbol{g}|, \quad \forall\left(\phi_{h}, \boldsymbol{u}_{h}\right) \in Y_{h} .
$$

Proof. It proceeds as in the proof of Lemma 3.2 Indeed, employing the same arguments used to derive 3.16 , we deduce that

$$
\left|\mathscr{C}_{\phi_{h}, \boldsymbol{u}_{h}}\left(\widetilde{\phi}_{h}, \widetilde{\psi}_{h}\right)\right| \leqslant \vartheta_{2}\left|\widetilde{\phi}_{h}\right|_{1, \Omega}\left|\widetilde{\psi}_{h}\right|_{1, \Omega}+\left\|\widetilde{\phi}_{h}\right\|_{L^{2} \tilde{q}(\Omega)}\left\|\boldsymbol{u}_{h}\right\|_{\mathbf{L}^{2} \tilde{p}(\Omega)}\left|\widetilde{\psi}_{h}\right|_{1, \Omega}+\beta\left\|\widetilde{\phi}_{h}\right\|_{0, \Omega}\left\|\widetilde{\psi}_{h}\right\|_{0, \Omega},
$$

where $\tilde{p}, \widetilde{q} \in[1,+\infty)$ are such that $1 / \widetilde{p}+1 / \widetilde{q}=1$. Thus, choosing $\widetilde{p}$ such that $2 \widetilde{p}=s$, with $s>2$, it readily follows that

$$
2 \widetilde{q}:=\frac{2 s}{s-2}>1
$$

We can then use (4.3), and the continuity of the injection $\mathrm{i}_{s}: \mathrm{H}^{1}(\Omega) \rightarrow \mathrm{L}^{\frac{2 s}{s-2}}(\Omega)$ with constant $\mathrm{C}_{s}$ (since for $2 \mathrm{D}$ this injection is compact) to deduce the existence of a positive constant $\|\mathscr{C}\|$ depending on $\vartheta_{2}, \beta, r$ and $\mathrm{C}_{S}$, such that

$$
\left|\mathscr{C}_{\phi_{h}, u_{h}}\left(\widetilde{\phi}_{h}, \widetilde{\psi}_{h}\right)\right| \leqslant\|\mathscr{C}\|\left\|\widetilde{\phi}_{h}\right\|_{1, \Omega}\left\|\widetilde{\psi}_{h}\right\|_{1, \Omega} \quad \forall \widetilde{\phi}_{h}, \widetilde{\psi}_{h} \in \mathrm{H}_{\Gamma_{0}}^{1}(\Omega),
$$

which proves that $\mathscr{C}_{\phi_{h}, \boldsymbol{u}_{h}}$ is bounded independently of $\phi_{h}$ and $\boldsymbol{u}_{h}$. On the other hand, we can apply the same argument to handle the trilinear term on the right-hand side of (4.11) in the derivation of (4.13). And employing estimates 2.5) and 2.8, we find that for each $\widetilde{\phi}_{h} \in \mathrm{H}_{\Gamma_{0}}^{1}(\Omega)$, there holds

$$
\begin{aligned}
\mathscr{C}_{\phi_{h}, \boldsymbol{u}_{h}}\left(\widetilde{\phi}_{h}, \widetilde{\phi}_{h}\right) & \geqslant \vartheta_{1}\left|\widetilde{\phi}_{h}\right|_{1, \Omega}^{2}-\left\|\widetilde{\phi}_{h}\right\|_{\mathrm{L}^{2 s / s-2}(\Omega)}\left\|\boldsymbol{u}_{h}\right\|_{\mathbf{L}^{s}(\Omega)}\left|\widetilde{\phi}_{h}\right|_{1, \Omega}+\beta\left\|\widetilde{\phi}_{h}\right\|_{0, \Omega}^{2} \geqslant\left(\vartheta_{1}-\mathrm{C}_{s} c_{p} r\right)\left|\widetilde{\phi}_{h}\right|_{1, \Omega}^{2} \\
& \geqslant \frac{\vartheta_{1}}{2}\left|\widetilde{\phi}_{h}\right|_{1, \Omega}^{2} \geqslant \frac{\vartheta_{1}}{2 c_{p}^{2}}\left\|\widetilde{\phi}_{h}\right\|_{1, \Omega}^{2},
\end{aligned}
$$

which proves that $\mathscr{C}_{\phi_{h}, u_{h}}$ is elliptic on $\mathrm{H}_{h}^{\phi} \times \mathrm{H}_{h}^{\phi}$, with the same constant $\widetilde{\alpha}$ from Lemma 3.2 In addition, the fact that $\left\|\mathscr{H}_{\phi}\right\|$ is bounded independently of $\phi$ (cf. Proof of Lemma 3.2, confirms the same upper bound for $\left\|\mathscr{H}_{\phi_{h}}\right\|_{\left(\mathrm{H}_{h}^{\phi}\right)^{\prime}}$. The rest of the proof is a straightforward application of Lax-Milgram's Lemma.

REMARK 4.1 The manipulation of the term $\beta\left\|\widetilde{\phi}_{h}\right\|_{0, \Omega}^{2}$ (in the derivation of the ellipticity of $\mathscr{C}_{\phi_{h}, \boldsymbol{u}_{h}}$ ) is done analogously as described at the end of the proof of Lemma 3.2 .

We end this section with an hypothesis of uniform boundedness on the operator $\mathbf{S}_{h}^{\text {flow }}$, which will be required to guarantee that the operator $\mathbf{T}_{h}$ is well-defined and continuous within a certain ball.

HypOTHESis 4.1 There exists $s>2$ such that the operator $\mathbf{S}_{h}^{\text {flow }}:\left(\mathrm{H}_{h}^{\phi},\|\cdot\|_{1, \Omega}\right) \rightarrow\left(\mathbf{X}_{h},\|\cdot\|_{s, \Omega}\right)(c f$. 4.5) is uniformly bounded, that is

$$
\left\|\mathbf{S}_{h}^{\text {flow }}\left(\phi_{h}\right)\right\|_{\mathbf{L}^{s}(\Omega)} \leqslant \widetilde{C}_{s}\left\|\phi_{h}\right\|_{1, \Omega} \quad \forall \phi_{h} \in \mathrm{H}_{h}^{\phi},
$$

where $\widetilde{C}_{s}$ is a positive constant independent of $h$.

Estimate (4.14) will be needed in the proof of Lemma 4.3 below. More specifically, it is required to bound an expression of the form $\left\|\mathbf{S}_{h}^{\text {flow }}\left(\mathbf{S}_{h}^{\operatorname{adv}}(\cdot)\right)\right\|_{\mathbf{L}^{s}(\Omega)}$ in terms of $\left\|\mathbf{S}_{h}^{\text {adv }}(\cdot)\right\|_{1, \Omega}$, which in turn is bounded by data $(c f$. 4.10). The hypothesis will also be required to handle the expression $\left\|\mathbf{S}_{h}^{\text {flow }}\left(\mathbf{S}_{h}^{\text {adv }}\left(\phi_{h}\right)\right)-\mathbf{S}_{h}^{\text {flow }}\left(\mathbf{S}_{h}^{\operatorname{adv}}\left(\varphi_{h}\right)\right)\right\|_{\mathbf{L}^{s}(\Omega)}$ when deriving the Lipschitz continuity of $\mathbf{T}_{h}$. Even if a rigorous proof for (4.14) can be quite difficult to obtain, in Section 6 we will provide numerical evidence that such $\widetilde{C}_{s}$ exists and that its is independent of $h$. 


\subsection{Solvability of the fixed-point equation}

We now aim to show the solvability of (4.2) by analysing the equivalent fixed-point equation (4.8). To this end, we proceed to verify the hypotheses of the Brouwer fixed-point theorem (Ciarlet. 2013. Theorem 9.9-2).

We start by defining the following set

$$
\mathbf{W}_{h}:=\left\{\left(\phi_{h}, \boldsymbol{u}_{h}\right) \in \mathrm{H}_{h}^{\phi} \times \mathbf{X}_{h}:\left\|\left|\left(\phi_{h}, \boldsymbol{u}_{h}\right) \|\right| \leqslant r\right\},\right.
$$

where $r$ was previously fixed in 4.3 , and

$$
\left|\left\|\left(\phi_{h}, \boldsymbol{u}_{h}\right)|| \mid:=\right\| \phi_{h}\left\|_{1, \Omega}+\right\| \boldsymbol{u}_{h}\left\|_{\star}=\right\| \phi_{h}\left\|_{1, \Omega}+\right\| \boldsymbol{u}_{h}\left\|_{\operatorname{div}, \Omega}+\right\| \boldsymbol{u}_{h} \|_{\mathbf{L}^{s}(\Omega)} .\right.
$$

LEMMA 4.3 Let $\mathbf{W}_{h}$ be as in 4.15, and assume that the data $g, f_{\mathrm{B}}$ and $\boldsymbol{f}_{\mathrm{D}}$ are sufficiently small so that

$$
\left(\widetilde{C}_{s}+C_{1}\right) \gamma_{2}|\Omega|^{1 / 2}|\boldsymbol{g}|+C_{1} C_{2} \gamma_{2}|\Omega|^{1 / 2}|\boldsymbol{g}|\left\{\left\|\boldsymbol{f}_{\mathrm{B}}\right\|_{\infty, \Omega_{\mathrm{B}}}+\left\|\boldsymbol{f}_{\mathrm{D}}\right\|_{\infty, \Omega_{\mathrm{D}}}\right\} \leqslant r .
$$

Then $\mathbf{T}_{h}\left(\mathbf{W}_{h}\right) \subseteq \mathbf{W}_{h}$

Proof. Given $\left(\phi_{h}, \boldsymbol{u}_{h}\right) \in \mathbf{W}_{h}$, we get from (4.7), and the estimates (4.14), (4.9), and (4.10), that

$$
\begin{aligned}
\left\|\mid \mathbf{T}_{h}\left(\phi_{h}, \boldsymbol{u}_{h}\right)\right\|\|=\| & \left\|\left(\mathbf{S}_{h}^{\mathrm{adv}}\left(\phi_{h}, \boldsymbol{u}_{h}\right), \mathbf{S}_{h}^{\text {flow }}\left(\mathbf{S}_{h}^{\text {adv }}\left(\phi_{h}, \boldsymbol{u}_{h}\right)\right)\right)\right\| \| \\
& =\left\|\mathbf{S}_{h}^{\text {adv }}\left(\phi_{h}, \boldsymbol{u}_{h}\right)\right\|_{1, \Omega}+\left\|\mathbf{S}_{h}^{\text {flow }}\left(\mathbf{S}_{h}^{\text {adv }}\left(\phi_{h}, \boldsymbol{u}_{h}\right)\right)\right\|_{\operatorname{div}, \Omega}+\left\|\mathbf{S}_{h}^{\text {flow }}\left(\mathbf{S}_{h}^{\text {adv }}\left(\phi_{h}, \boldsymbol{u}_{h}\right)\right)\right\|_{\mathbf{L}^{s}(\Omega)} \\
& \leqslant\left(\widetilde{C}_{s}+C_{1}\right) \gamma_{2}|\Omega|^{1 / 2}|\boldsymbol{g}|+C_{1} C_{2} \gamma_{2}|\Omega|^{1 / 2}|\boldsymbol{g}|\left\{\left\|\boldsymbol{f}_{\mathrm{B}}\right\|_{\infty, \Omega_{\mathrm{B}}}+\left\|\boldsymbol{f}_{\mathrm{D}}\right\|_{\infty, \Omega_{\mathrm{D}}}\right\},
\end{aligned}
$$

and hence, employing the condition 4.16, we conclude that $\mathbf{T}_{h}\left(\phi_{h}, \boldsymbol{u}_{h}\right) \in \mathbf{W}_{h}$.

The continuity of $\mathbf{T}_{h}$ will be proved in the following two lemmas, where we derive Lipschitz type-estimates for $\mathbf{S}_{h}^{\text {flow }}$ and $\mathbf{S}_{h}^{\text {adv }}$.

LEMma 4.4 Let $C_{2}$ be the constant given in Lemma 4.1 Then, there holds

$$
\left\|\mathbf{S}_{h}^{\text {flow }}\left(\phi_{h}\right)-\mathbf{S}_{h}^{\text {flow }}\left(\varphi_{h}\right)\right\|_{\operatorname{div}, \Omega} \leqslant C_{2}\left\|\phi_{h}-\varphi_{h}\right\|_{0, \Omega}\left\{\left\|\boldsymbol{f}_{\mathrm{B}}\right\|_{\infty, \Omega_{\mathrm{B}}}+\left\|\boldsymbol{f}_{\mathrm{D}}\right\|_{\infty, \Omega_{\mathrm{D}}}\right\} \quad \forall \phi_{h}, \varphi_{h} \in \mathrm{H}_{h}^{\phi} .
$$

Proof. Given $\phi_{h}, \varphi_{h} \in \mathrm{H}_{h}^{\phi}$, we let $\vec{u}_{h}:=\left(\boldsymbol{u}_{h}^{\mathrm{B}}, \boldsymbol{\omega}_{h}^{\mathrm{B}}, \boldsymbol{u}_{h}^{\mathrm{D}}\right) \in \mathbf{H}_{h}, \vec{p}_{h}:=\left(p_{h}^{\mathrm{B}}, p_{h}^{\mathrm{D}}, \lambda_{h}\right) \in \mathbf{Q}_{0, h}$ and $\vec{w}_{h}=\left(\boldsymbol{w}_{h}^{\mathrm{B}}, \boldsymbol{x}_{h}^{\mathrm{B}}, \boldsymbol{w}_{h}^{\mathrm{D}}\right) \in$ $\mathbf{H}_{h}, \vec{r}_{h}:=\left(r_{h}^{\mathrm{B}}, r_{h}^{\mathrm{D}}, \chi_{h}\right) \in \mathbf{Q}_{0, h}$ be the solution of 4.6, so that $\boldsymbol{u}_{h}=\boldsymbol{u}_{h}^{\mathrm{B}}+\boldsymbol{u}_{h}^{\mathrm{D}}=: \mathbf{S}_{h}^{\text {flow }}\left(\phi_{h}\right)$ and $\boldsymbol{w}_{h}=\boldsymbol{w}_{h}^{\mathrm{B}}+\boldsymbol{w}_{h}^{\mathrm{D}}=$ : $\mathbf{S}_{h}^{\text {flow }}\left(\varphi_{h}\right)$. Then, employing the linearity of the forms $\mathscr{A}$ and $\mathscr{B}$, we deduce from 4.6 that

$$
\begin{aligned}
\mathscr{A}\left(\overrightarrow{\boldsymbol{u}}_{h}-\overrightarrow{\boldsymbol{w}}_{h}, \overrightarrow{\boldsymbol{v}}_{h}\right)+\mathscr{B}\left(\overrightarrow{\boldsymbol{v}}_{h}, \vec{p}_{h}-\vec{r}_{h}\right) & =\mathscr{F}_{\phi_{h}-\varphi_{h}}\left(\overrightarrow{\boldsymbol{v}}_{h}\right) & & \forall \overrightarrow{\boldsymbol{v}}_{h}:=\left(\boldsymbol{v}_{h}^{\mathrm{B}}, z_{h}^{\mathrm{B}}, \boldsymbol{v}_{h}^{\mathrm{D}}\right) \in \mathbf{H}, \\
\mathscr{B}\left(\overrightarrow{\boldsymbol{u}}_{h}-\overrightarrow{\boldsymbol{w}}_{h}, \vec{q}_{h}\right) & =0 & & \forall \vec{q}_{h}:=\left(q_{h}^{\mathrm{B}}, q_{h}^{\mathrm{D}}, \xi_{h}\right) \in \mathbf{Q}_{0, h} .
\end{aligned}
$$

And since $\mathbf{S}_{h}^{\text {flow }}\left(\phi_{h}-\varphi_{h}\right)=\mathbf{S}_{h}^{\text {flow }}\left(\phi_{h}\right)-\mathbf{S}_{h}^{\text {flow }}\left(\varphi_{h}\right)$, the bound 4.17) follows directly from estimate 4.9.

LEMMA 4.5 Let $L_{f_{\mathrm{bk}}}, L_{\vartheta}$, and $\widetilde{\alpha}$ be the constants given in 2.7, 2.6, and Lemma 3.2 respectively. Then,

$$
\begin{aligned}
\left\|\mathbf{S}_{h}^{\mathrm{adv}}\left(\phi_{h}, \boldsymbol{u}_{h}\right)-\mathbf{S}_{h}^{\mathrm{adv}}\left(\varphi_{h}, \boldsymbol{w}_{h}\right)\right\|_{1, \Omega} \leqslant & \frac{1}{\widetilde{\alpha}}\left\{L_{f_{\mathrm{bk}}}|\boldsymbol{g}|\left\|\phi_{h}-\varphi_{h}\right\|_{0, \Omega}+L_{\vartheta}\left\|\nabla \mathbf{S}_{h}^{\mathrm{adv}}\left(\phi_{h}, \boldsymbol{u}_{h}\right)\right\|_{\mathrm{L}^{4}(\Omega)}\left\|\phi_{h}-\varphi_{h}\right\|_{\mathrm{L}^{4}(\Omega)}\right. \\
& \left.+\left\|\mathbf{S}_{h}^{\mathrm{adv}}\left(\phi_{h}, \boldsymbol{u}_{h}\right)\right\|_{\mathrm{L}^{2 \tilde{q}}(\Omega)}\left\|\boldsymbol{u}_{h}-\boldsymbol{w}_{h}\right\|_{\mathbf{L}^{2 \tilde{p}}(\Omega)}\right\},
\end{aligned}
$$

$\forall\left(\phi_{h}, \boldsymbol{u}_{h}\right),\left(\varphi_{h}, \boldsymbol{w}_{h}\right) \in \mathrm{H}_{h}^{\phi} \times \mathbf{Z}_{h}^{r}$, and where $\widetilde{p}, \widetilde{q} \in[1,+\infty)$ are such that $1 / \widetilde{p}+1 / \widetilde{q}=1$.

Proof. Given $\left(\phi_{h}, \boldsymbol{u}_{h}\right),\left(\varphi_{h}, \boldsymbol{w}_{h}\right)$ as stated, we let $\widetilde{\phi}_{h}:=\mathbf{S}_{h}^{\mathrm{adv}}\left(\phi_{h}, \boldsymbol{u}_{h}\right)$ and $\widetilde{\varphi}_{h}:=\mathbf{S}_{h}^{\mathrm{adv}}\left(\varphi_{h}, \boldsymbol{w}_{h}\right)$, that is

$$
\mathscr{C}_{\phi_{h}, \boldsymbol{u}_{h}}\left(\widetilde{\phi}_{h}, \widetilde{\psi}_{h}\right)=\mathscr{H}_{\phi_{h}}\left(\widetilde{\psi}_{h}\right) \quad \text { and } \quad \mathscr{C}_{\varphi_{h}, \boldsymbol{w}_{h}}\left(\widetilde{\varphi}_{h}, \widetilde{\psi}_{h}\right)=\mathscr{H}_{\varphi_{h}}\left(\widetilde{\psi}_{h}\right) \quad \forall \widetilde{\psi}_{h} \in \mathrm{H}_{h}^{\phi} .
$$

Next we proceed analogously as in the proof of Lemma 3.4 We apply Cauchy-Schwarz's inequality, the Lipschitz-continuity estimates (2.6)-(2.7), and Hölder's inequality to the second term on the right-hand side for the discrete version of (3.24). For the last term we use an $\mathrm{L}^{4}-\mathrm{L}^{4}-\mathrm{L}^{2}$ argument. This gives

$$
\begin{gathered}
\widetilde{\alpha}\left\|\widetilde{\phi}_{h}-\widetilde{\varphi}_{h}\right\|_{1, \Omega}^{2} \leqslant\{ \\
\left.L_{f_{\mathrm{bk}}}|\boldsymbol{g}|\left\|\phi_{h}-\varphi_{h}\right\|_{0, \Omega}+\left\|\widetilde{\varphi}_{h}\right\|_{\mathrm{L}^{2 \widetilde{q}}(\Omega)}\left\|\boldsymbol{u}_{h}-\boldsymbol{w}_{h}\right\|_{\mathbf{L}^{2 \tilde{p}}(\Omega)}\right\}\left|\widetilde{\phi}_{h}-\widetilde{\varphi}_{h}\right|_{1, \Omega} \\
+L_{\vartheta}\left\|\phi_{h}-\varphi_{h}\right\|_{\mathrm{L}^{4}(\Omega)}\left\|\nabla \widetilde{\varphi}_{h}\right\|_{\mathrm{L}^{4}(\Omega)}\left|\widetilde{\phi}_{h}-\widetilde{\varphi}_{h}\right|_{1, \Omega} .
\end{gathered}
$$


Then, since the elements of $\mathrm{H}_{h}^{\phi}$ are piecewise polynomials, it follows that $\left\|\nabla \widetilde{\varphi}_{h}\right\|_{\mathrm{L}^{4}(\Omega)}<+\infty$, and hence the foregoing equation yields 4.18.

We now can establish a Lipschitz continuity type-estimate for $\mathbf{T}_{h}$.

LEMMA 4.6 Given $r>0$, and $\mathbf{W}_{h}$ as in 4.15), assume the condition (4.16). Then, there exist constants $C_{3}, C_{4}, C_{5}>0$, depending only upon $L_{f_{\mathrm{bk}}}, L_{\vartheta}, \widetilde{\alpha}, C_{2}, \widetilde{C}_{s}(c f$. 2.7), 2.6, Lemma 3.2 Lemma 4.4. (4.14) and the data, such that, for all $\left(\phi_{h}, \boldsymbol{u}_{h}\right),\left(\varphi_{h}, \boldsymbol{w}_{h}\right) \in \mathbf{W}_{h}$, there holds

$$
\begin{aligned}
\left\|\mid \mathbf{T}_{h}\left(\phi_{h}, \boldsymbol{u}_{h}\right)-\mathbf{T}_{h}\left(\varphi_{h}, \boldsymbol{w}_{h}\right)\right\| \| \leqslant & C_{3}\left\|\phi_{h}-\varphi_{h}\right\|_{0, \Omega}+C_{4}\left\|\nabla \mathbf{S}_{h}^{\operatorname{adv}}\left(\phi_{h}, \boldsymbol{u}_{h}\right)\right\|_{\mathrm{L}^{4}(\Omega)}\left\|\phi_{h}-\varphi_{h}\right\|_{\mathrm{L}^{4}(\Omega)} \\
& +C_{5}\left\|\mathbf{S}_{h}^{\operatorname{adv}}\left(\phi_{h}, \boldsymbol{u}_{h}\right)\right\|_{\mathrm{L}^{2 \widetilde{q}}(\Omega)}\left\|\boldsymbol{u}_{h}-\boldsymbol{w}_{h}\right\|_{\mathbf{L}^{2 \tilde{p}}(\Omega)},
\end{aligned}
$$

where $\widetilde{p}, \widetilde{q} \in[1,+\infty)$ are such that $1 / \widetilde{p}+1 / \widetilde{q}=1$.

Proof. It suffices to recall from Section 4.1 that

$$
\mathbf{T}_{h}\left(\psi_{h}, \boldsymbol{v}_{h}\right):=\left(\mathbf{S}_{h}^{\mathrm{adv}}\left(\psi_{h}, \boldsymbol{v}_{h}\right), \mathbf{S}_{h}^{\text {flow }}\left(\mathbf{S}_{h}^{\mathrm{adv}}\left(\psi_{h}, \boldsymbol{v}_{h}\right)\right)\right) \quad \forall\left(\psi_{h}, \boldsymbol{v}_{h}\right) \in Y_{h},
$$

and then apply estimate 4.14, and Lemmas 4.4 and 4.5

Consequently, from the foregoing Lemma, choosing $2 \widetilde{p}$ and $2 \widetilde{q}$ as in the proof of Lemma 4.2 that is $2 \widetilde{p}=s$ and hence $2 \widetilde{q}:=\frac{2 s}{s-2}>1$; and employing the continuous injection i : $\mathrm{H}^{1}(\Omega) \rightarrow \mathrm{L}^{4}(\Omega)$, the continuity of $\mathbf{T}_{h}$ is derived. Thanks to Brouwer's fixed-point theorem (Ciarlet 2013, Theorem 9.9-2) and Lemmas 4.3 and 4.6 we establish the main result of this section.

THEOREM 4.1 Under the assumptions of Lemma 4.3, the Galerkin scheme 4.2] has at least one solution $\left(\overrightarrow{\boldsymbol{u}}_{h}, \vec{p}_{h}, \phi_{h}\right) \in \mathbf{H}_{h} \times \mathbf{Q}_{0, h} \times \mathrm{H}_{h}^{\phi}$ with $\left(\phi_{h}, \boldsymbol{u}_{h}\right) \in \mathbf{W}_{h}$. Moreover

$$
\begin{aligned}
\left\|\phi_{h}\right\|_{1, \Omega} & \leqslant C_{1} \gamma_{2}|\Omega|^{1 / 2}|\boldsymbol{g}|, \\
\left\|\left(\overrightarrow{\boldsymbol{u}}_{h}, \vec{p}_{h}\right)\right\|_{\mathbf{H} \times \mathbf{Q}_{0}} & \leqslant C_{1} C_{2} \gamma_{2}|\Omega|^{1 / 2}|\boldsymbol{g}|\left\{\left\|\boldsymbol{f}_{\mathrm{B}}\right\|_{\infty, \Omega_{\mathrm{B}}}+\left\|\boldsymbol{f}_{\mathrm{D}}\right\|_{\infty, \Omega_{\mathrm{D}}}\right\},
\end{aligned}
$$

where $C_{1}, C_{2}$, and $\gamma_{2}$, are the constants provided by Lemmas 4.1 and 4.2 as well as 2.5 , respectively.

REMARK 4.2 The extension to 3D case of our discrete fixed-point analysis is based on a new range for the parameter $s$ in (4.3), Lemma 4.2, and Hypothesis 4.14 More precisely, one needs now to take $s>3$ in 4.3 to guarantee the compactness (and hence the continuity) of the injection $\mathrm{i}_{s}: \mathrm{H}^{1}(\Omega) \rightarrow \mathrm{L}^{\frac{3 s}{s-3}}(\Omega)$. This is crucial in the proof of Lemma 4.2

\section{A priori error estimate}

Given $(\overrightarrow{\boldsymbol{u}}, \vec{p}, \phi) \in \mathbf{H} \times \mathbf{Q}_{0} \times \mathrm{H}_{\Gamma_{0}}^{1}(\Omega)$ with $\phi \in \mathrm{W}$, and $\left(\overrightarrow{\boldsymbol{u}}_{h}, \vec{p}_{h}, \phi_{h}\right) \in \mathbf{H}_{h} \times \mathbf{Q}_{0, h} \times \mathrm{H}_{h}^{\phi}$ with $\left(\phi_{h}, \boldsymbol{u}_{h}\right) \in \mathbf{W}_{h}$, we aim to derive suitable a priori error estimates. To this end, we first observe from 3.7 and 4.2 , that the above problems can be rewritten as follows:

$$
\begin{aligned}
& (B D)\left\{\begin{array}{rll}
\mathscr{A}(\overrightarrow{\boldsymbol{u}}, \overrightarrow{\boldsymbol{v}})+\mathscr{B}(\overrightarrow{\boldsymbol{v}}, \vec{p})= & \mathscr{F}_{\phi}(\overrightarrow{\boldsymbol{v}}) & \forall \overrightarrow{\boldsymbol{v}} \in \mathbf{H}, \\
\mathscr{B}(\overrightarrow{\boldsymbol{u}}, \vec{q})=0 & 0 & \forall \vec{q} \in \mathbf{Q}_{0},
\end{array}\right. \\
& \left(B D_{h}\right)\left\{\begin{aligned}
\mathscr{A}\left(\overrightarrow{\boldsymbol{u}}_{h}, \overrightarrow{\boldsymbol{v}}_{h}\right)+\mathscr{B}\left(\overrightarrow{\boldsymbol{v}}_{h}, \vec{p}_{h}\right)= & \mathscr{F}_{\phi_{h}}\left(\overrightarrow{\boldsymbol{v}}_{h}\right) & \forall \overrightarrow{\boldsymbol{v}}_{h} \in \mathbf{H}_{h}, \\
\mathscr{B}\left(\overrightarrow{\boldsymbol{u}}_{h}, \vec{q}_{h}\right)= & 0 & \forall \vec{q}_{h} \in \mathbf{Q}_{0, h},
\end{aligned}\right.
\end{aligned}
$$

and

$$
\begin{array}{rlrl}
(T) \mathscr{C}_{\boldsymbol{u}}(\phi, \psi) & =\mathscr{H}_{\phi}(\psi) \quad \forall \psi \in \mathrm{H}_{\Gamma_{0}}^{1}(\Omega), \\
\left(T_{h}\right) \quad \mathscr{C}_{\boldsymbol{u}_{h}}\left(\phi_{h}, \psi_{h}\right) & =\mathscr{H}_{\phi_{h}}\left(\psi_{h}\right) & \forall \psi_{h} \in \mathrm{H}_{h}^{\phi} .
\end{array}
$$

LEMmA 5.1 There exists $C_{\mathrm{B}, \mathrm{D}}>0$, independent of $h$, such that

$$
\begin{aligned}
& \left\|(\overrightarrow{\boldsymbol{u}}, \vec{p})-\left(\overrightarrow{\boldsymbol{u}}_{h}, \vec{p}_{h}\right)\right\|_{\mathbf{H} \times \mathbf{Q}_{0}} \\
& \quad \leqslant C_{\mathrm{B}, \mathrm{D}}\left\{\operatorname{dist}\left(\overrightarrow{\boldsymbol{u}}, \mathbf{H}_{h}\right)+\operatorname{dist}\left(\vec{p}, \mathbf{Q}_{0, h}\right)+\left\|\phi-\phi_{h}\right\|_{1, \Omega}\left(\left\|\boldsymbol{f}_{\mathrm{B}}\right\|_{\infty, \Omega_{\mathrm{B}}}+\left\|\boldsymbol{f}_{\mathrm{D}}\right\|_{\infty, \Omega_{\mathrm{D}}}\right)\right\} .
\end{aligned}
$$


Proof. We derive a Strang-type error estimate for $(B D)$ and $\left(B D_{h}\right)$. For this we proceed similarly as in (Gatica et al. 2016. Section 4) and deduce the existence of a positive constant $C_{\mathrm{B}, \mathrm{D}}$, independent of $h$, such that

$$
\left\|(\overrightarrow{\boldsymbol{u}}, \vec{p})-\left(\overrightarrow{\boldsymbol{u}}_{h}, \vec{p}_{h}\right)\right\|_{\mathbf{H} \times \mathbf{Q}_{0}} \leqslant C_{\mathrm{B}, \mathrm{D}}\left\{\operatorname{dist}\left(\overrightarrow{\boldsymbol{u}}, \mathbf{H}_{h}\right)+\operatorname{dist}\left(\vec{p}, \mathbf{Q}_{0, h}\right)+\left\|\mathscr{F}_{\phi}-\mathscr{F}_{\phi_{h}}\right\|_{\mathbf{H}_{h}^{\prime}}\right\} .
$$

Next, according to the definition of $\mathscr{F}_{\phi}$ and $\mathscr{F}_{\phi_{h}}(c f$. 3.3), and applying Cauchy-Schwarz's inequality, we easily deduce that

$$
\left\|\mathscr{F}_{\phi}-\mathscr{F}_{\phi_{h}}\right\|_{\mathbf{H}_{h}^{\prime}} \leqslant\left\|\phi-\phi_{h}\right\|_{0, \Omega}\left(\left\|f_{\mathrm{B}}\right\|_{\infty, \Omega_{\mathrm{B}}}+\left\|\boldsymbol{f}_{\mathrm{D}}\right\|_{\infty, \Omega_{\mathrm{D}}}\right) .
$$

The proof is completed after replacing $[5.3]$ into $[5.2]$.

We now derive a Céa estimate for the term $\left\|\phi-\phi_{h}\right\|_{1, \Omega}$, focusing in the $2 \mathrm{D}$ case. For notational convenience we define the following constants, all independent of the data $g, f_{\mathrm{B}}$, and $f_{\mathrm{D}}$,

$$
K_{1}:=C_{\mathbf{S}^{\text {adv }}}\left\{L_{f_{\mathrm{bk}}}+L_{\vartheta} \mathrm{C}_{\delta} \mathrm{C}_{\delta}^{*} \widehat{C}_{\mathbf{S}^{\mathrm{adv}}}(r) \gamma_{2}|\Omega|^{1 / 2}\right\}, \quad K_{2}:=C_{\mathrm{S}^{\mathrm{adv}}}\left(r \mathrm{C}_{s}+\beta+\vartheta_{2}\right)+1, \quad \text { and } \quad K_{3}=C_{\mathbf{S}^{\mathrm{adv}}} .
$$

Here $\widehat{C}_{\mathbf{S}^{\text {adv }}}(r)$ and $C_{\mathrm{S}^{\text {adv }}}$ are given in 3.20 and 3.15 , and $C_{S}, \mathrm{C}_{\delta}, \mathrm{C}_{\delta}^{*}$ are the boundedness constants of the continuous injections

$$
\mathrm{i}_{s}: \mathrm{H}^{1}(\Omega) \longrightarrow \mathrm{L}^{\frac{2 s}{s-2}}(\Omega), \quad \mathrm{i}: \mathrm{H}^{1}(\Omega) \longrightarrow \mathrm{L}^{2 / \delta}(\Omega), \quad \mathrm{i}_{\delta}: \mathrm{H}^{\delta}(\Omega) \longrightarrow \mathrm{L}^{\delta^{*}}(\Omega),
$$

respectively, where $s>2, \delta \in(0,1)$, and $\delta^{*}:=2 /(1-\delta)$. In addition, in order to handle a term in the derivation of the Céa estimate for $\left\|\phi-\phi_{h}\right\|_{1, \Omega}$, we will further assume that $\phi \in \mathrm{L}^{\infty}(\Omega)$.

LEMma 5.2 Assume that $\phi \in \mathrm{H}^{1}(\Omega) \cap \mathrm{L}^{\infty}(\Omega)$, and that the data $g$ satisfy

$$
K_{1}|\boldsymbol{g}| \leqslant \frac{1}{2} .
$$

Then, there holds

$$
\left\|\phi-\phi_{h}\right\|_{1, \Omega} \leqslant 2 K_{2} \operatorname{dist}\left(\phi, H_{h}^{\phi}\right)+2 K_{3}\|\phi\|_{\infty, \Omega}\left\|\boldsymbol{u}-\boldsymbol{u}_{h}\right\|_{0, \Omega} .
$$

Proof. Triangle inequality immediately gives

$$
\left\|\phi-\phi_{h}\right\|_{1, \Omega} \leqslant\left\|\phi-\varphi_{h}\right\|_{1, \Omega}+\left\|\phi_{h}-\varphi_{h}\right\|_{1, \Omega} \quad \forall \varphi_{h} \in \mathrm{H}_{h}^{\phi} .
$$

Then we employ the ellipticity of the bilinear form $\mathscr{C}_{\phi_{h}, \boldsymbol{u}_{h}}$ with constant $\widetilde{\alpha}$, and subsequently add and subtract one term, we deduce that

$$
\begin{aligned}
\widetilde{\alpha}\left\|\phi_{h}-\varphi_{h}\right\|_{1, \Omega}^{2} & \leqslant \mathscr{C}_{\phi_{h}, \boldsymbol{u}_{h}}\left(\phi_{h}-\varphi_{h}, \phi_{h}-\varphi_{h}\right) \\
& \leqslant\left|\mathscr{H}_{\phi_{h}}\left(\phi_{h}-\varphi_{h}\right)-\mathscr{H}_{\phi}\left(\phi_{h}-\varphi_{h}\right)\right|+\left|\mathscr{C}_{\phi, \boldsymbol{u}}\left(\phi, \phi_{h}-\varphi_{h}\right)-\mathscr{C}_{\phi_{h}, \boldsymbol{u}_{h}}\left(\varphi_{h}, \phi_{h}-\varphi_{h}\right)\right| .
\end{aligned}
$$

Next, according to 3.10, and applying Cauchy-Schwarz's inequality, we get

$$
\left|\mathscr{H}_{\phi_{h}}\left(\phi_{h}-\varphi_{h}\right)-\mathscr{H}_{\phi}\left(\phi_{h}-\varphi_{h}\right)\right| \leqslant L_{f_{\mathrm{bk}}}|\boldsymbol{g}|\left\|\phi-\phi_{h}\right\|_{0, \Omega}\left|\phi_{h}-\varphi_{h}\right|_{1, \Omega} .
$$

In turn, adding and subtracting suitable expressions, and then applying Hölder's inequality, the upper bound of $\vartheta\left(c f\right.$. (2.5) ) and its Lipschitz continuity (cf. (2.6)), and using $\phi \in \mathrm{L}^{\infty}(\Omega)$, we find that

$$
\begin{aligned}
& \left|\mathscr{C}_{\phi, \boldsymbol{u}}\left(\phi, \phi_{h}-\varphi_{h}\right)-\mathscr{C}_{\phi_{h}, \boldsymbol{u}_{h}}\left(\varphi_{h}, \phi_{h}-\varphi_{h}\right)\right| \\
& \quad \leqslant \quad L_{\vartheta}\left\|\phi-\phi_{h}\right\|_{\mathrm{L}^{2 q}(\Omega)}\|\nabla \phi\|_{\mathrm{L}^{2 p}(\Omega)}\left|\phi_{h}-\varphi_{h}\right|_{1, \Omega}+\vartheta_{2}\left|\phi-\varphi_{h}\right|_{1, \Omega}\left|\phi_{h}-\varphi_{h}\right|_{1, \Omega} \\
& \quad+\|\phi\|_{\infty, \Omega}\left\|\boldsymbol{u}-\boldsymbol{u}_{h}\right\|_{0, \Omega}\left|\phi_{h}-\varphi_{h}\right|_{1, \Omega}+\left\|\phi-\varphi_{h}\right\|_{\mathrm{L}^{2 \tilde{q}}(\Omega)}\left\|\boldsymbol{u}_{h}\right\|_{\mathbf{L}^{2 \tilde{p}}(\Omega)}\left|\phi_{h}-\varphi_{h}\right|_{1, \Omega} \\
& \quad+\quad \beta\left\|\phi-\varphi_{h}\right\|_{0, \Omega}\left\|\phi_{h}-\varphi_{h}\right\|_{0, \Omega},
\end{aligned}
$$

where $p, q, \widetilde{p}, \widetilde{q} \in[1,+\infty)$ are such that $1 / p+1 / q=1$ and $1 / \widetilde{p}+1 / \widetilde{q}=1$. In this way, choosing $2 p$ and $2 q$ as in 3.17, $2 \widetilde{p}$ and $2 \widetilde{q}$ as in (4.12), and applying the continuous embeddings $\mathrm{i}, \mathrm{i}_{\delta}, \mathrm{i}_{s}$, estimate 3.20 , and the fact that $\left\|\boldsymbol{u}_{h}\right\|_{\mathbf{L}^{s}(\Omega)} \leqslant r$, it follows from 5.10) that

$$
\begin{aligned}
& \left|\mathscr{C}_{\phi, \boldsymbol{u}}\left(\phi, \phi_{h}-\varphi_{h}\right)-\mathscr{C}_{\phi_{h}, \boldsymbol{u}_{h}}\left(\varphi_{h}, \phi_{h}-\varphi_{h}\right)\right| \\
& \quad \leqslant \quad L_{\vartheta} \mathrm{C}_{\delta} \mathrm{C}_{\delta}^{*} \widehat{C}_{\mathbf{S}^{\mathrm{adv}}}(r) \gamma_{2}|\Omega|^{1 / 2}|\boldsymbol{g}|\left\|\phi-\phi_{h}\right\|_{1, \Omega}\left\|\phi_{h}-\varphi_{h}\right\|_{1, \Omega} \\
& \quad+\quad \vartheta_{2}\left\|\phi-\varphi_{h}\right\|_{1, \Omega}\left\|\phi_{h}-\varphi_{h}\right\|_{1, \Omega}+r \mathrm{C}_{s}\left\|\phi-\varphi_{h}\right\|_{1, \Omega}\left\|\phi_{h}-\varphi_{h}\right\|_{1, \Omega} \\
& \quad+\|\phi\|_{\infty, \Omega}\left\|\boldsymbol{u}-\boldsymbol{u}_{h}\right\|_{0, \Omega}\left\|\phi_{h}-\varphi_{h}\right\|_{1, \Omega}+\beta\left\|\phi-\varphi_{h}\right\|_{1, \Omega}\left\|\phi_{h}-\varphi_{h}\right\|_{1, \Omega} .
\end{aligned}
$$


Finally, we replace (5.9) and (5.11) into (5.8). The resulting estimate is combined with (5.7), and after recalling (from the proof of Lemma 3.2 that $\widetilde{\alpha}=C_{\mathbf{S}^{\text {adv }}}^{-}$and applying several algebraic manipulations, we find that

$$
\left\|\phi-\phi_{h}\right\|_{1, \Omega} \leqslant K_{1}|\boldsymbol{g}|\left\|\phi-\phi_{h}\right\|_{1, \Omega}+K_{2}\left\|\phi-\varphi_{h}\right\|_{1, \Omega}+\|\phi\|_{\infty, \Omega}\left\|\boldsymbol{u}-\boldsymbol{u}_{h}\right\|_{0, \Omega} \quad \forall \varphi_{h} \in \mathrm{H}_{h}^{\phi} .
$$

Then, we use the data constraint 5.5 , and take the infimum $\varphi_{h} \in \mathrm{H}_{h}^{\phi}$, yielding (5.2).

REMARK 5.1 Extending the proof of Lemma 5.2 to the 3D case requires to modify the choice of parameters $\delta$ and $\delta^{*}$, and hence $2 p$ and $2 q$, similarly as in (3.17). In turn, as discussed in Remark 4.2 one needs to take $s>3$ and then choose $2 \widetilde{p}$ and $2 \widetilde{q}$ analogously as in 4.12 . This permits to invoke the continuous injection $\mathrm{i}_{s}: \mathrm{H}^{1}(\Omega) \rightarrow \mathrm{L}^{\frac{3 s}{s-3}}(\Omega)$.

We now combine Lemmas 5.1 and 5.2 to derive the Céa estimate for the total error $\left\|(\overrightarrow{\boldsymbol{u}}, \vec{p})-\left(\overrightarrow{\boldsymbol{u}}_{h}, \vec{p}_{h}\right)\right\|_{\mathbf{H} \times \mathbf{Q}_{0}+}$ $\left\|\phi-\phi_{h}\right\|_{1, \Omega}$. Replacing the estimate for $\left\|\phi-\phi_{h}\right\|_{1, \Omega}$ given by $\sqrt{5.6}$ into the second term on the right-hand side of (5.1), we find that

$$
\begin{aligned}
\left\|(\overrightarrow{\boldsymbol{u}}, \vec{p})-\left(\overrightarrow{\boldsymbol{u}}_{h}, \vec{p}_{h}\right)\right\|_{\mathbf{H} \times \mathbf{Q}_{0} \leqslant} & C_{\mathrm{B}, \mathrm{D}}\left\{\operatorname{dist}\left(\overrightarrow{\boldsymbol{u}}, \mathbf{H}_{h}\right)+\operatorname{dist}\left(\vec{p}, \mathbf{Q}_{0, h}\right)\right\}+\widetilde{K} \operatorname{dist}\left(\boldsymbol{\phi}, \mathrm{H}_{h}^{\phi}\right) \\
& +2 C_{\mathrm{B}, \mathrm{D}}\|\boldsymbol{\phi}\|_{\infty, \Omega}\left(\left\|\boldsymbol{f}_{\mathrm{B}}\right\|_{\infty, \Omega_{\mathrm{B}}}+\left\|\boldsymbol{f}_{\mathrm{D}}\right\|_{\infty, \Omega_{\mathrm{D}}}\right)\left\|\boldsymbol{u}-\boldsymbol{u}_{h}\right\|_{0, \Omega},
\end{aligned}
$$

where

$$
\widetilde{K}:=2 K_{2} C_{\mathrm{B}, \mathrm{D}}\left(\left\|f_{\mathrm{B}}\right\|_{\infty, \Omega_{\mathrm{B}}}+\left\|f_{\mathrm{D}}\right\|_{\infty, \Omega_{\mathrm{D}}}\right) .
$$

Assuming now that the data $f_{\mathrm{B}}$ and $f_{\mathrm{D}}$ satisfy

$$
C_{\mathrm{B}, \mathrm{D}}\|\phi\|_{\infty, \Omega}\left\|\boldsymbol{f}_{\mathrm{B}}\right\|_{\infty, \Omega_{\mathrm{B}}}+C_{\mathrm{B}, \mathrm{D}}\|\phi\|_{\infty, \Omega}\left\|\boldsymbol{f}_{\mathrm{D}}\right\|_{\infty, \Omega_{\mathrm{D}}} \leqslant \frac{1}{4},
$$

we can conclude that

$$
\left\|(\overrightarrow{\boldsymbol{u}}, \vec{p})-\left(\overrightarrow{\boldsymbol{u}}_{h}, \vec{p}_{h}\right)\right\|_{\mathbf{H} \times \mathbf{Q}_{0}} \leqslant 2 C_{\mathrm{B}, \mathrm{D}}\left\{\operatorname{dist}\left(\overrightarrow{\boldsymbol{u}}, \mathbf{H}_{h}\right)+\operatorname{dist}\left(\vec{p}, \mathbf{Q}_{0, h}\right)\right\}+2 \widetilde{K} \operatorname{dist}\left(\phi, \mathrm{H}_{h}^{\phi}\right) .
$$

THEOREM 5.1 Assume that $\phi \in \mathrm{H}^{1}(\Omega) \cap \mathrm{L}^{\infty}(\Omega)$, and that the data $f_{\mathrm{B}}, f_{\mathrm{D}}$ and $\boldsymbol{g}$ are sufficiently small so that $K_{1}|\boldsymbol{g}| \leqslant \frac{1}{2}$ and 5.12 hold. Then, there exists a positive constant $C$ independent of $h$, such that

$$
\left\|(\overrightarrow{\boldsymbol{u}}, \vec{p})-\left(\overrightarrow{\boldsymbol{u}}_{h}, \vec{p}_{h}\right)\right\|_{\mathbf{H} \times \mathbf{Q}_{0}}+\left\|\phi-\phi_{h}\right\|_{1, \Omega} \leqslant C\left\{\operatorname{dist}\left(\overrightarrow{\boldsymbol{u}}, \mathbf{H}_{h}\right)+\operatorname{dist}\left(\vec{p}, \mathbf{Q}_{0, h}\right)+\operatorname{dist}\left(\phi, \mathrm{H}_{h}^{\phi}\right)\right\} .
$$

Proof. It follows straightforwardly from the Céa estimates [5.13) and 5.6.

The following theorem provides the rate of convergence of our Galerkin scheme 4.2.

THEOREM 5.2 Let $\mathbf{H}_{h}:=\mathbf{H}_{h}^{\mathrm{B}} \times \mathbf{H}_{0, h}^{\mathrm{B}} \times \mathbf{H}_{h}^{\mathrm{D}}, \mathbf{Q}_{h, 0}:=\mathrm{Q}_{h, 0}^{\mathrm{B}} \times \mathrm{Q}_{h}^{\mathrm{D}} \times \mathrm{Q}_{\tilde{h}}^{\Sigma}$ and $\mathrm{H}_{h}^{\phi}$, be the subspaces specified in Section 4 In addition, let $(\overrightarrow{\boldsymbol{u}}, \vec{p}, \boldsymbol{\phi}):=\left(\left(\boldsymbol{u}_{\mathrm{B}}, \boldsymbol{\omega}_{\mathrm{B}}, \boldsymbol{u}_{\mathrm{D}}\right),\left(p_{\mathrm{B}}, p_{\mathrm{D}}, \lambda\right), \boldsymbol{\phi}\right) \in \mathbf{H} \times \mathbf{Q}_{0} \times \mathrm{H}_{\Gamma_{0}}^{1}(\Omega)$ and $\left(\overrightarrow{\boldsymbol{u}}_{h}, \vec{p}_{h}, \boldsymbol{\phi}_{h}\right)$ $:=\left(\left(\boldsymbol{u}_{h}^{\mathrm{B}}, \boldsymbol{\omega}_{h}^{\mathrm{B}}, \boldsymbol{u}_{h}^{\mathrm{D}}\right),\left(p_{h}^{\mathrm{B}}, p_{h}^{\mathrm{D}}, \lambda_{\tilde{h}}\right), \boldsymbol{\phi}_{h}\right) \in \mathbf{H}_{h} \times \mathbf{Q}_{0, h} \times \mathrm{H}_{h}^{\phi}$ be the solutions of the continuous and discrete problems (3.7) and (4.2), respectively. Assume that $\boldsymbol{u}_{\star} \in \mathbf{H}^{l}\left(\Omega_{\star}\right), \operatorname{div} \boldsymbol{u}_{\star} \in \mathrm{H}^{l}\left(\Omega_{\star}\right), p_{\star} \in \mathrm{H}^{l}\left(\Omega_{\star}\right)$ where $\star \in\{\mathrm{B}, \mathrm{D}\}$, $\boldsymbol{\omega}_{\mathrm{B}} \in \mathbf{H}^{l}\left(\mathbf{c u r l} ; \Omega_{\mathrm{B}}\right), \lambda \in \mathrm{H}^{1 / 2+l}(\Sigma)$ and $\phi \in \mathrm{H}_{\Gamma_{0}}^{1+l}(\Omega)$, for some $l \in(0,1)($ when $n=2)$ or $l \in(1 / 2,1)$ (when $n=3)$. Then, there exists $C>0$ and $\widetilde{C}>0$ independent of $h$ and $\tilde{h}$ such that

$$
\begin{aligned}
& \left\|(\overrightarrow{\boldsymbol{u}}, \vec{p})-\left(\overrightarrow{\boldsymbol{u}}_{h}, \vec{p}_{h}\right)\right\|_{\mathbf{H} \times \mathbf{Q}_{0}}+\left\|\boldsymbol{\phi}-\phi_{h}\right\|_{1, \Omega} \\
& \quad \leqslant C h^{\min \{l, k+1\}}\left\{\left\|\boldsymbol{u}_{\mathrm{B}}\right\|_{l, \Omega_{\mathrm{B}}}+\left\|\operatorname{div}\left(\boldsymbol{u}_{\mathrm{B}}\right)\right\|_{l, \Omega_{\mathrm{B}}}+\left\|\boldsymbol{\omega}_{\mathrm{B}}\right\|_{\mathbf{H}^{l}\left(\mathbf{c u r l} ; \Omega_{\mathrm{B}}\right)}+\left\|\boldsymbol{u}_{\mathrm{D}}\right\|_{l, \Omega_{\mathrm{D}}}\right. \\
& \left.\quad+\left\|\operatorname{div}\left(\boldsymbol{u}_{\mathrm{D}}\right)\right\|_{l, \Omega_{\mathrm{D}}}+\left\|p_{\mathrm{B}}\right\|_{l, \Omega_{\mathrm{B}}}+\left\|p_{\mathrm{D}}\right\|_{l, \Omega_{\mathrm{D}}}+\|\boldsymbol{\phi}\|_{1+l, \Omega}\right\}+\widetilde{C} \tilde{h}^{l}\|\lambda\|_{l+1 / 2, \Sigma} .
\end{aligned}
$$

Proof. It follows directly from the Céa estimate (5.14) and the approximation properties of the finite element spaces (see Alvarez et al. 2016b. Section 4.2.2 and Ciarlet 1978).

\section{Numerical examples}




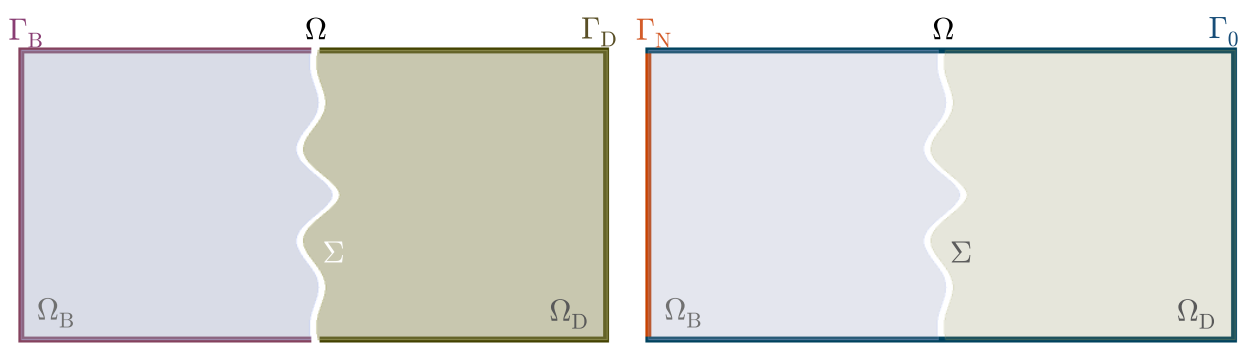

FIG. 6.1. Test 1. Domain and boundary configuration for the Brinkman-Darcy problem, where $\partial \Omega=\Gamma_{\mathrm{B}} \cup \Gamma_{\mathrm{D}}$ (left panel), and for the transport equation where $\partial \Omega=\Gamma_{\mathrm{N}} \cup \Gamma_{0}$ (right). The unit normal vector on the interface points towards $\Omega_{\mathrm{B}}$.

TEST 1. We begin this section with an accuracy test, where we construct smooth solutions satisfying (2.1)2.3 on $\Omega=(0,2) \times(0,1)$. The Brinkman and Darcy domains are on the left and right parts of $\Omega$, respectively, and are separated by the curved interface $\Sigma$ defined by the parameterisation

$$
(0,1) \ni t \mapsto\left(x_{1}, x_{2}\right)=(1+0.15[1 / 2-|t-1 / 2|] \cos (6 \pi t-3 \pi), t),
$$

on which the normal vector is considered pointing to $\Omega_{\mathrm{B}}$. The proposed exact solutions are given by

$$
\begin{aligned}
& \boldsymbol{u}=\left(\begin{array}{c}
\sin \left(\pi x_{1}\right) \cos \left(\pi x_{2}\right) \\
-\cos \left(\pi x_{1}\right) \sin \left(\pi x_{2}\right)
\end{array}\right), \quad \boldsymbol{u}_{\mathrm{B}}=\left.\boldsymbol{u}\right|_{\Omega_{\mathrm{B}}}, \quad \boldsymbol{u}_{\mathrm{D}}=\left.\boldsymbol{u}\right|_{\Omega_{\mathrm{D}}}, \quad \boldsymbol{\omega}_{\mathrm{B}}=2 \pi \sin \left(\pi x_{1}\right) \sin \left(\pi x_{2}\right), \\
& p=\left(x_{1}-1 / 2\right)\left(x_{2}-1 / 2\right), \quad p_{\mathrm{B}}=\left.p\right|_{\Omega_{\mathrm{B}}}, \quad p_{\mathrm{D}}=\left.p\right|_{\Omega_{\mathrm{D}}}, \quad \phi=\frac{5}{2} x_{1}^{2}\left(2-x_{1}\right) x_{2}\left(1-x_{2}\right),
\end{aligned}
$$

from which the necessary forcing, boundary, and source terms are generated. The concentration-dependent functions are $\vartheta(\phi)=\phi+(1-c \phi)^{2}$, and $f_{\mathrm{bk}}(\phi)=c \phi(1-c \phi)^{2}$, and the remaining physical parameters assume the values $c=1 / 2, f_{\mathrm{B}}=(1,0)^{2}, f_{\mathrm{D}}=(0.1,0)^{\mathrm{t}}, \mathbb{K}_{\mathrm{B}}=0.05, \mathbb{K}_{\mathrm{D}}=0.01, \beta=\left.0.4 \chi\right|_{\Omega_{\mathrm{B}}}+\left.0.1 \chi\right|_{\Omega_{\mathrm{D}}}, \boldsymbol{k}=(0,-1)^{\mathrm{t}}$. We recall that two different splittings of the domain boundary $\partial \Omega$ are assumed. First, the distribution of the Brinkman and Darcy boundaries follows the sketch presented in the left panel of Figure 6.1 According to (2.4), on $\Gamma_{\mathrm{B}}$ we set slip velocities $\boldsymbol{u}_{\mathrm{B}} \cdot \boldsymbol{n}=0$ and zero tangential vorticity (in this $2 \mathrm{D}$ case, it translates to fix the scalar vorticity to zero), but on $\Sigma$ we prescribe the vorticity by its exact solution. Normal Darcy velocities are fixed on $\Gamma_{\mathrm{D}}: \boldsymbol{u}_{\mathrm{D}} \cdot \boldsymbol{n}=0$. Secondly, by construction, the concentration normal flux is zero on the left side of $\Gamma_{\mathrm{B}}$, which constitutes the Neumann boundary $\Gamma_{\mathrm{N}}$. The remainder of $\partial \Omega$ conforms the Dirichlet boundary $\Gamma_{0}$, where we impose $\phi=0$ (see Figure 6.1. right). Both domains are rendered with a small gap on the interface, for visualisation purposes.

As usual, to determine the convergence of the method we generate a sequence of successively refined triangulations of $\Omega$ (and conforming partitions for $\Omega_{\mathrm{B}}, \Omega_{\mathrm{D}}$ and $\Sigma$ ) and proceed to compute errors and they decay rates according to

$$
\begin{aligned}
e\left(\boldsymbol{u}_{\mathrm{B}}\right) & =\left\|\boldsymbol{u}_{\mathrm{B}}-\boldsymbol{u}_{\mathrm{B} h}\right\|_{\mathrm{div}, \Omega_{\mathrm{B}}}, e\left(\boldsymbol{\omega}_{\mathrm{B}}\right)=\left\|\boldsymbol{\omega}_{\mathrm{B}}-\boldsymbol{\omega}_{\mathrm{B} h}\right\|_{\operatorname{curl}, \Omega_{\mathrm{B}}}, e\left(\boldsymbol{u}_{\mathrm{D}}\right)=\left\|\boldsymbol{u}_{\mathrm{D}}-\boldsymbol{u}_{\mathrm{D} h}\right\|_{\mathrm{div}, \Omega_{\mathrm{D}}}, \\
e\left(p_{\mathrm{B}}\right) & =\left\|p_{\mathrm{B}}-p_{\mathrm{B} h}\right\|_{0, \Omega_{\mathrm{B}}}, e\left(p_{\mathrm{D}}\right)=\left\|p_{\mathrm{D}}-p_{\mathrm{D} h}\right\|_{0, \Omega_{\mathrm{D}}}, e(\lambda)=\left\|\lambda-\lambda_{h}\right\|_{0, \Sigma}\left\|\lambda-\lambda_{h}\right\|_{1, \Sigma}, \\
e(\phi) & =\left\|\phi-\phi_{h}\right\|_{1, \Omega}, r(\cdot)=-2 \log (e(\cdot) / \hat{e}(\cdot))[\log (N / \hat{N})]^{-1},
\end{aligned}
$$

where $e$ and $\hat{e}$ denote errors produced on two consecutive meshes associated with schemes with $N$ and $\hat{N}$ D.o.f. (degrees of freedom), respectively. The results are collected in Figure 6.2, where we plot the decaying of individual errors with the meshsize, for the lowest-order scheme. All panels indicate an $O(h)$ convergence, as anticipated by Theorem 5.2. We point out that an average of seven Picard steps (accounting for the coupling between the Brinkman-Darcy and transport problems) are required to reach the stopping tolerance of $1 e-6$, whereas an average of three Newton steps are sufficient to achieve convergence of the inner linearisation step (with a tolerance of $1 e-7$ ) for the nonlinear advection-diffusion problem. We also portray the approximate solutions obtained with the proposed method on a fine mesh (see Figure 6.3 .

An important assumption in the solvability analysis was an additional regularity for the discrete velocity, as stated in Hypothesis 4.1 Even if proving this assumption can be very difficult, we can at least provide numerical evidence of its validity for the finite element spaces we employ here. For instance, taking the regularity index as $s=2.5$, and obtaining approximate solutions in the same refinement levels as mentioned above (whose 

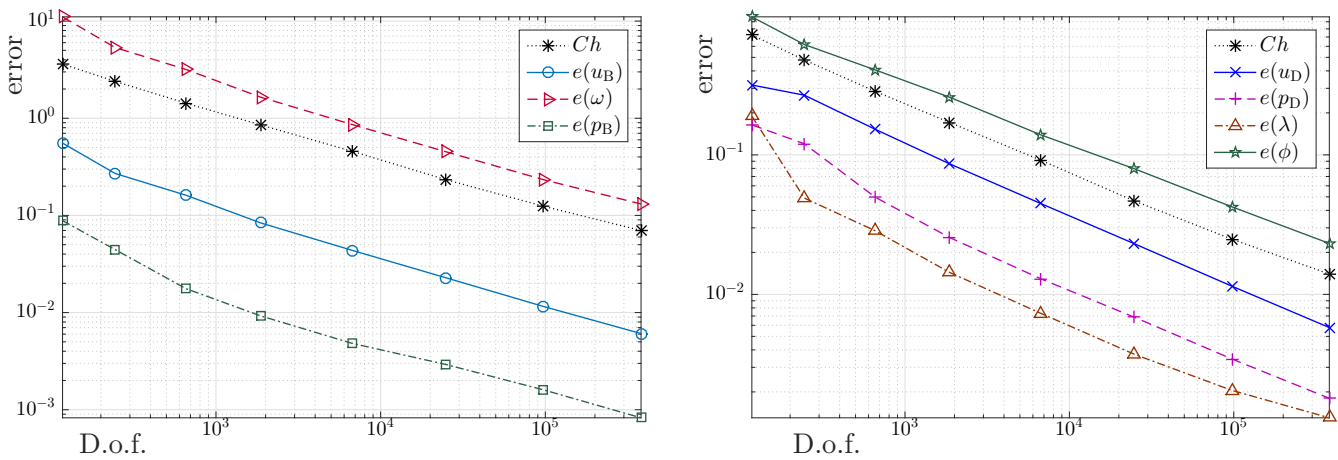

FIG. 6.2. Test 1. Convergence history for the lowest-order approximation of the coupled Brinkman-Darcy-Transport problem.

error history is depicted in Figure 6.2, the values reported in Table 6.1 are produced. The ratios between the $\mathbf{L}^{s}$-norm of the discrete velocity and the $\mathrm{H}^{1}$-norm of the concentration are tabulated in the last column and they suggest that the constant $\widetilde{C}_{S}$ is indeed uniform. These values correspond to the approximate solutions after convergence of the Picard algorithm. Taking the last mesh refinement as an example, we can also see in Table 6.2 how the constant $\widetilde{C}_{s}$ evolves throughout the nine fixed-point steps.

\begin{tabular}{|l|c|c|c|c|c|c|c|c|c|c|}
\hline D.o.f. & $h$ & $e\left(\boldsymbol{u}_{\mathrm{B}}\right)$ & $r\left(\boldsymbol{u}_{\mathrm{B}}\right)$ & $e\left(\boldsymbol{u}_{\mathrm{D}}\right)$ & $r\left(\boldsymbol{u}_{\mathrm{D}}\right)$ & $e(\phi)$ & $r(\phi)$ & $\left\|\mathbf{S}^{\text {flow }}\left(\phi_{h}\right)\right\|_{\mathbf{L}^{s}(\Omega)}$ & $\left\|\phi_{h}\right\|_{1, \Omega}$ & $\widetilde{C}_{s}$ \\
\hline 111 & 0.723 & 0.562 & - & 0.371 & - & 0.823 & - & 0.644 & 1.791 & 0.5219 \\
237 & 0.479 & 0.269 & 1.786 & 0.267 & 0.791 & 0.622 & 0.678 & 0.920 & 1.825 & 0.5700 \\
664 & 0.285 & 0.160 & 0.997 & 0.155 & 1.045 & 0.407 & 0.819 & 1.022 & 1.890 & 0.5695 \\
1920 & 0.170 & 0.082 & 1.308 & 0.086 & 1.149 & 0.264 & 0.837 & 0.903 & 1.894 & 0.5689 \\
6625 & 0.099 & 0.043 & 1.160 & 0.044 & 1.117 & 0.161 & 0.975 & 1.082 & 1.907 & 0.5670 \\
24907 & 0.048 & 0.022 & 0.931 & 0.022 & 0.950 & 0.083 & 1.011 & 1.080 & 1.911 & 0.5656 \\
97907 & 0.023 & 0.011 & 0.956 & 0.011 & 0.962 & 0.022 & 1.041 & 1.079 & 1.911 & 0.5643 \\
382115 & 0.014 & 0.006 & 1.066 & 0.006 & 1.161 & 0.012 & 1.008 & 1.078 & 1.911 & 0.5643 \\
\hline
\end{tabular}

Table 6.1. Test 1. Convergence history for velocity and concentration, and illustration of Hypothesis 4.1 for the lowest-order scheme. Here we have used $s=2.5$.

\begin{tabular}{|l|l|l|l|l|l|l|l|l|}
\hline 0.55012 & 0.56600 & 0.56450 & 0.56435 & 0.56435 & 0.56435 & 0.56435 & 0.56435 & 0.56435 \\
\hline
\end{tabular}

Table 6.2. Test 1. Illustration of Hypothesis 4.1 Variation of the constant $\widetilde{C}_{s}$ for the lowest-order scheme on the last refinement level.

TEST 2. Our second example addresses the applicability of the formulation and the associated numerical scheme in the simulation of groundwater flow, where we have followed the setup adopted in Test 4.1 of Çeşmelioğlu et al. (2010) (see also Alvarez et al. (2016b)). The computational domain now corresponds to the rectangle $\Omega=(0,12) \times(0,6)$ (in square meters), where the Brinkman domain (with a maximum height of $4 \mathrm{~m}$ ) is on the top and the Darcy subdomain (with a maximum height of $2.25 \mathrm{~m}$ ) on the bottom. The subdomains are separated by a step-shaped polygonal interface (see a sketch in the top-left panel of Figure 6.4 where we also depict sample triangular meshes). We consider $\mu=1 \mathrm{~Pa} \cdot \mathrm{s}, \beta=\left.0.7 \chi\right|_{\Omega_{\mathrm{B}}}+\left.0.07 \chi\right|_{\Omega_{\mathrm{D}}}$, and the permeabilities are again isotropic and assume the values $\mathbb{K}_{\mathrm{B}}=1 e-6 \mathrm{~m}^{2}, \mathbb{K}_{\mathrm{D}}=1 e-7 \mathrm{~m}^{2}$ Normal velocities are imposed everywhere on $\partial \Omega$. On the top segment of $\Gamma_{\mathrm{B}}$ and in all $\Gamma_{\mathrm{D}}$ these are simply zero, whereas on the left and right sides of the Brinkman domain we prescribe the parabolic profiles

$$
\boldsymbol{u}_{\mathrm{B}} \cdot \boldsymbol{n}=\frac{1}{4}(y-4)(y-8), \quad \text { and } \quad \boldsymbol{u}_{\mathrm{B}} \cdot \boldsymbol{n}=\frac{3}{16}(y-4)(8-y),
$$



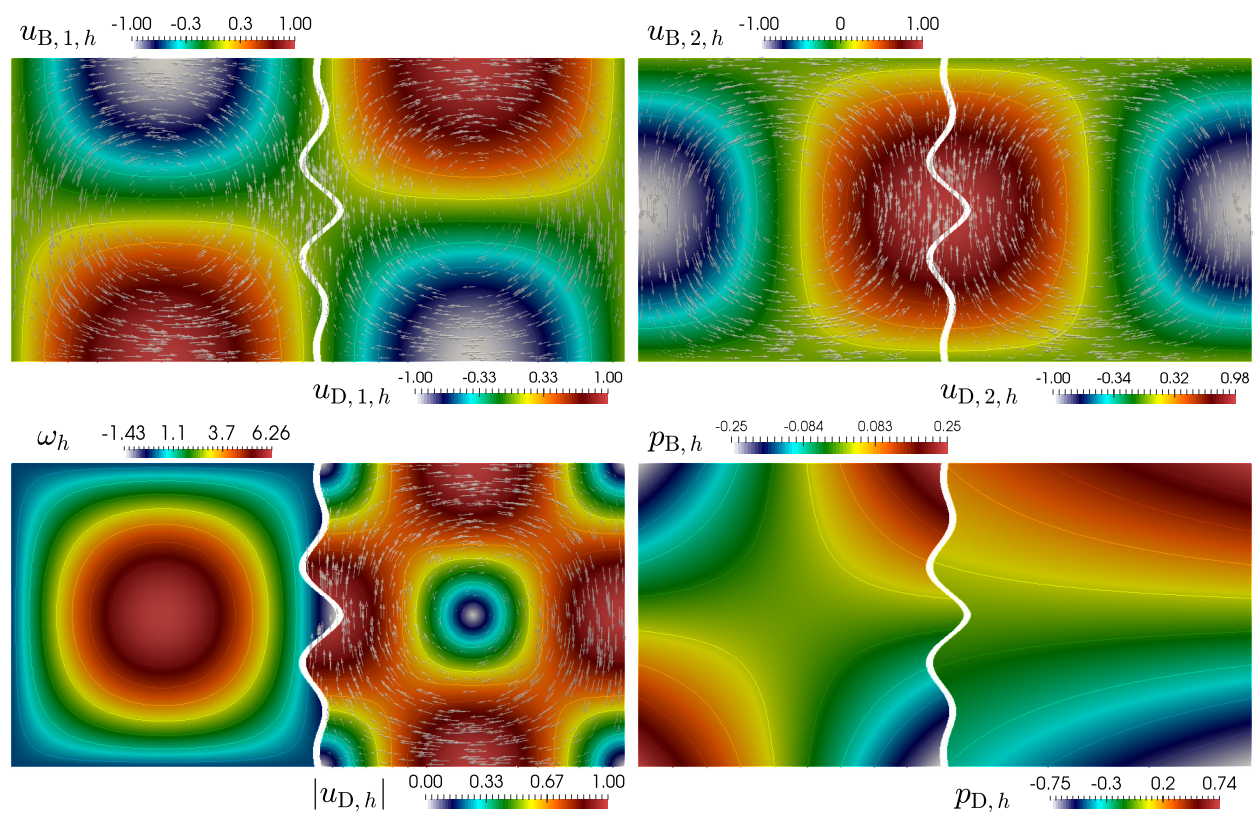
$u_{\mathrm{D}}$
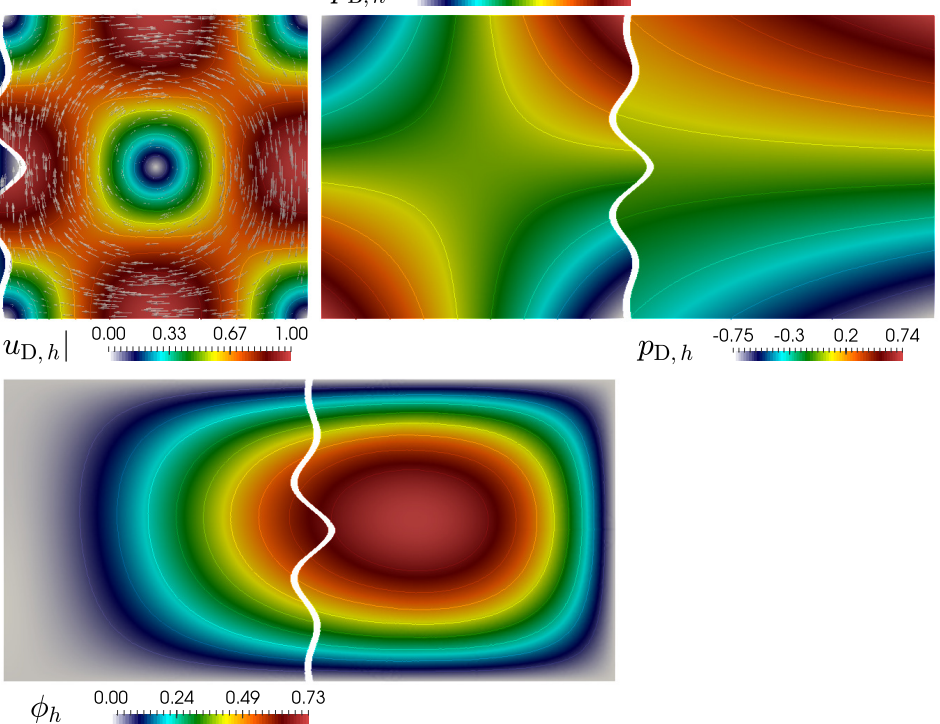

FIG. 6.3. Test 1. Approximate velocity components, vorticity, pressure, and concentration, rendered on both Brinkman and Darcy domains.

respectively, as well as the compatible vorticity $\boldsymbol{\omega}_{\mathrm{B}}=\frac{1}{2}(y-6)$ and $\boldsymbol{\omega}_{\mathrm{B}}=\frac{3}{8}(y-6)$, respectively. Regarding the transport equation, on the left side of the Brinkman domain (denoted by $\Gamma_{0}$ ) we impose a maximum solute concentration $\phi=\phi_{\max }=0.99$, whereas zero total flux is considered on $\Gamma_{\mathrm{N}}=\partial \Omega \backslash \Gamma_{0}$. The nonlinear diffusion assumes the form $\vartheta(\phi, \boldsymbol{u})=\exp \left(-\frac{1}{4} \phi\right)+0.01|\boldsymbol{u}|+\beta$ and the flux is simply linear $f_{\mathrm{bk}}(\phi)=0.001 \phi$. We take $\boldsymbol{k}=(0,-1)^{\mathrm{t}}$ and assume that an external source modulates the Brinkman flow (for instance due to rain, to wind, or to the leakage of contaminants through the top portion of the boundary, that represents the surface) so we use $\boldsymbol{f}_{\mathrm{B}}=(0.01,0.001)^{\mathrm{t}}$. We employ a second-order scheme resulting on a linear system of $1,517,352$ unknowns for the Brinkman-Darcy subproblem and 327,707 D.o.f. for the transport equation. Seven fixed-point iterations were needed to reach the desired tolerance and only two Newton steps were required for the convergence of the inner linearisation (probably due to the fact that the nonlinear diffusion is in this case milder than that used in Tests 1 and 3). The results are collected in Figure 6.4 which shows flow patterns as well as the solute entering the domain and starting to propagate towards the right.

TEST 3. We finalise this section presenting a 3D computation that illustrates the use of our method in the numerical simulation of filtering devices. Flow-rate conditions are taken similarly to those employed in Ervin et al. (2009); Anaya et al. (2015), namely a cylindrical geometry aligned with the $y$-axis, with varying cross section; where the Brinkman domain is the region with largest radius $(r=4 \mathrm{~cm}$ and length $L=6 \mathrm{~cm})$, and the Darcy domain constitutes the two other sections of the device (of radii $r=2 \mathrm{~cm}$ and $r=3 \mathrm{~cm}$ and lengths of $L=3 \mathrm{~cm}$ and $L=5.1 \mathrm{~cm}$, respectively). We assume that there is an inlet boundary belonging to $\Gamma_{\mathrm{B}}$ and an outlet disk at the end of the cylinder, on $\Gamma_{\mathrm{D}}$. 

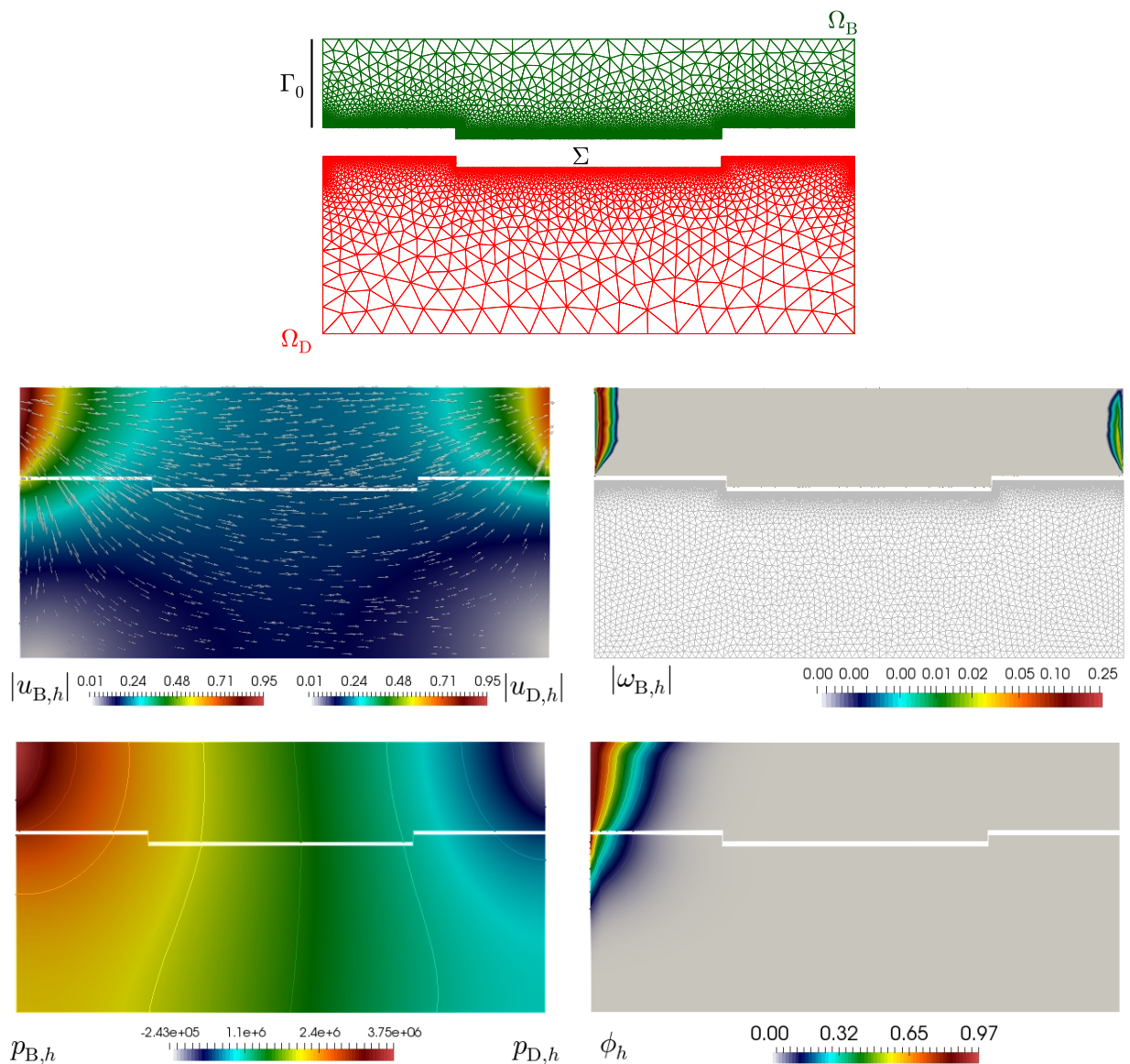

FIG. 6.4. Test 2. Sample mesh and domain configuration (top), and approximate velocity, vorticity magnitude, pressure, and solute concentration produced with a second-order scheme.

The flow is driven essentially by injection of fluid. A Poiseuille Brinkman velocity is prescribed at the inlet, as well a compatible vorticity

$$
\boldsymbol{u}_{\mathrm{B}} \cdot \boldsymbol{n}=2\left(1-\frac{1}{4}\left(x^{2}+z^{2}\right)\right), \quad \text { and } \quad \boldsymbol{\omega}_{\mathrm{B}} \times \boldsymbol{n}=\left(-2 x\left(1-\frac{1}{4}\left(x^{2}+z^{2}\right)\right), 0,-2 x\left(1-\frac{1}{4}\left(x^{2}+z^{2}\right)\right)\right)^{\mathrm{t}},
$$

whereas on the outlet boundary we impose a constant Darcy pressure $p_{\mathrm{D}}=p_{0}=0.1$. On the remainder of the domain boundary we set slip conditions for velocity (and zero tangential vorticity on the curved Brinkman boundary). For the transport equation we impose a constant concentration on the inlet and assume zero total flux everywhere else, therefore the inlet (the disc of radius $4 \mathrm{~m}$ and centred at the origin) is the boundary $\Gamma_{0}$ and the remainder of the boundary is $\Gamma_{\mathrm{N}}$. The interface conditions correspond to the ones stated in (2.4), and a depiction of the domain and boundary setup is presented in the first panel of Figure 6.5 The constitutive equations specifying the nonlinear diffusion $\vartheta$ and the unidirectional flux $f_{\mathrm{bk}}$ are simply taken as in Test 1 above, with $c=0.4$. Other model parameters are chosen as

$$
\begin{gathered}
\mu=0.01, \quad \mathbb{K}_{\mathrm{B}}=0.01, \quad \mathbb{K}_{\mathrm{D}}=0.00001, \quad \beta=\left\{\begin{array}{ll}
\frac{1}{2} & \text { in } \Omega_{\mathrm{B}}, \\
10 & \text { in } \Omega_{\mathrm{D}}
\end{array}, \quad \boldsymbol{f}_{\mathrm{D}}=(0,1,0)^{\mathrm{t}},\right. \\
\boldsymbol{f}_{\mathrm{B}}=\left(\exp (-x y)+x \exp \left(-x^{2}\right), \cos (\pi y)-y \exp \left(-y^{2}\right), x y z-z \exp \left(-z^{2}\right)\right)^{\mathrm{t}}, \quad \boldsymbol{k}=(0,1,0)^{\mathrm{t}},
\end{gathered}
$$

where we note that the hydraulic conductivity is discontinuous across the interface. The domain has been discretised with an unstructured tetrahedral mesh of 74,108 elements, and we have employed a first-order scheme. The approximate solutions are shown in the remaining panels of Figure 6.5 The first observation from the velocity streamlines is that the non-symmetric external force $f_{\mathrm{B}}$ rapidly disrupts the Poiseuille profile as the 


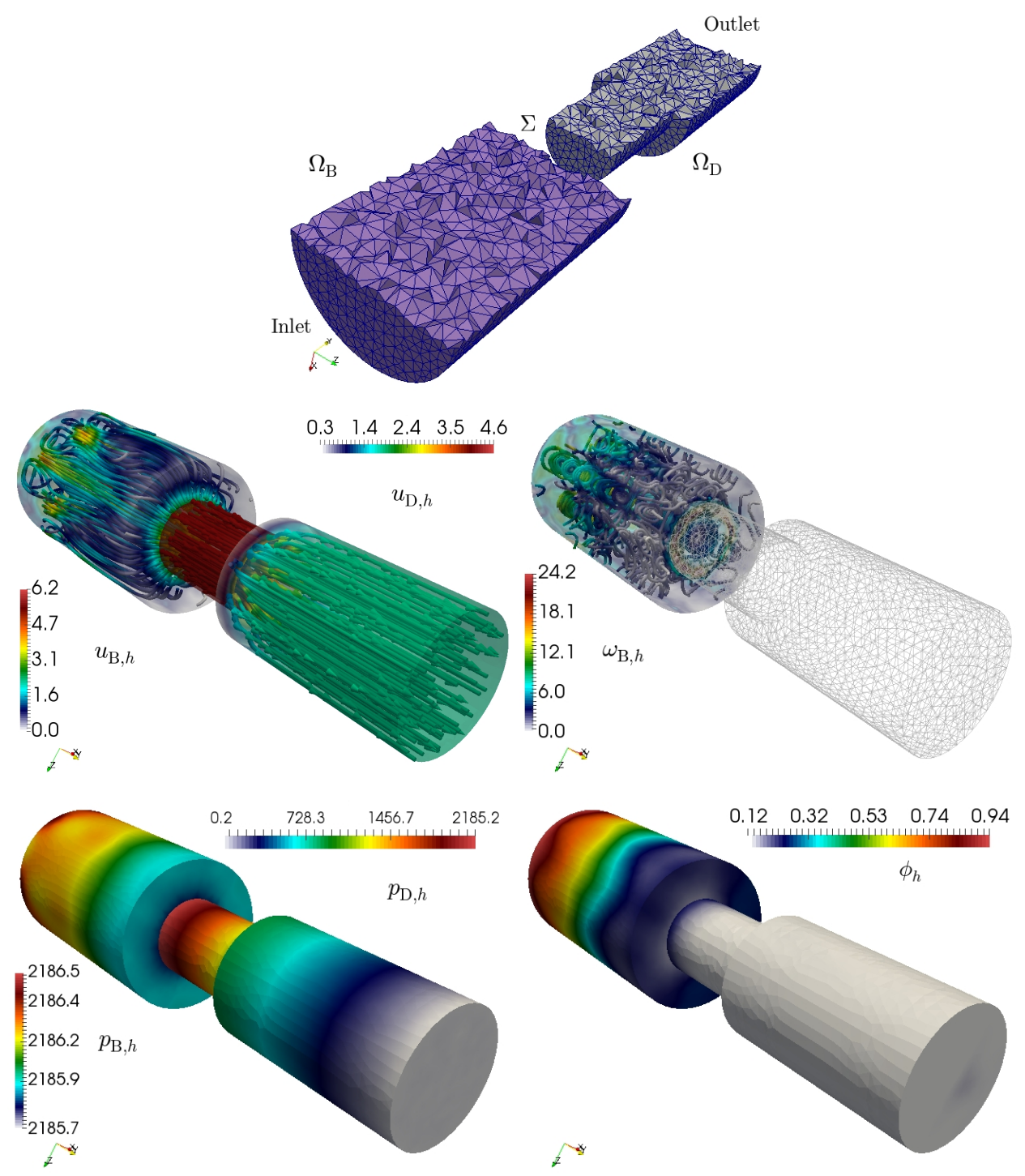

FIG. 6.5. Test 3. Sample (coarse) mesh and domain configuration (top), and approximate velocity, vorticity, pressure, and solute concentration produced with a first-order scheme.

flow moves away from the inlet. We can also see that the Lagrange multiplier enforces correctly the continuity of pressure across the interface but that there exists a very large Brinkman pressure and a large pressure drop is then seen in the Darcy domain. Also, the tangential components of vorticity slowly decrease when approaching the the interface. As the flow patterns stabilise due to the interfacial conditions, the propagation of concentration also becomes very uniform.

For this problem, the nonlinear coupling between flow and transport seems to be stronger than before, as the convergence of the Picard algorithm occurred after nine iterations and the inner Newton iterations for the transport problem converged after four steps.

Acknowledgement. We are grateful to Javier Almonacid (SFU) for stimulating discussions on interface fluid problems, and for providing an initial implementation of Test 2. We also acknowledge the constructive suggestions made by the managing Editor and by anonymous referees, which have significantly improved the presentation of this paper. 


\section{References}

Adams, R.A., Fournier, J.J.F., Sobolev Spaces. Academic Press. Elsevier Ltd., 2003.

Alvarez, M., Gatica, G.N., Ruiz-BAier, R., An augmented mixed-primal finite element method for a coupled flow-transport problem. ESAIM: Math. Model. Numer. Anal., 49 (2015), no. 5, 1399-1427.

Alvarez, M., Gatica, G.N., Ruiz-BAier, R., A mixed-primal finite element approximation of a sedimentation-consolidation system. M3AS: Math. Models Methods Appl. Sci., 26 (2016), no. 5, 867-900.

Alvarez, M., GatiCA, G.N., Ruiz-BAIER, R., Analysis of a vorticity-based fully-mixed formulation for the 3D Brinkman-Darcy problem. Comput. Methods Appl. Mech. Engrg., 307 (2016), 68-95.

Anaya, V., Mora, D., Reales, C., Ruiz-BAier, R., Stabilized mixed approximation of axisymmetric Brinkman flows. ESAIM: Math. Model. Numer. Anal., 49 (2015), no. 3, 855-874.

Anaya, V., Mora, D., Reales, C., Ruiz-BAier, R., Vorticity-pressure finite element formulations for the Brinkman-Darcy coupled problem. Numer. Methods Part. Diff. Eqns., 35 (2019), no. 2, 528-544.

AnAya, V., Mora, D., OYARzÚA, R., RUIZ-BAIER, R., A priori and a posteriori error analysis for a mixed scheme for the Brinkman problem. Numer. Math., 133 (2016), no. 4, 781-817.

ANAYA, V., MORA, D., RUIZ-BAIER, R., An augmented mixed finite element method for the vorticity-velocitypressure formulation of the Stokes equations. Comput. Methods Appl. Mech. Engrg., 267 (2013) 261-274.

ÇeşmelioĞLu, A., Chidyagwai, P., Rivière, B., Continuous and discontinuous finite element methods for coupled surface-subsurface flow and transport problems. Technical Report TR10-09. Available from scholarship.rice.edu.

ÇEŞMElioĞLU, A., Rivière, B., Existence of a weak solution for the fully coupled Navier-Stokes/Darcytransport problem. J. Diff. Eqns., 252 (2012), no. 7, 4138-4175.

Ciarlet, P., The Finite Element Method for Elliptic Problems. North-Holland, 1978.

CIARLET, P., Linear and Nonlinear Functional Analysis with Applications. Society for Industrial and Applied Mathematics, Philadelphia, PA, 2013.

Ehrhardt, M., Fuhrmann, J., Linke, A., A model of an electrochemical flow cell with porous layer. WIAS technical report No. 1437, (2009).

Ervin, V., Jenkins, E., Sun, S., Coupled generalized non-linear Stokes flow with flow through a porous media. SIAM J. Numer. Anal., 47 (2009), 929-952.

Ervin, V., Kubacki, M., Layton, W., Moraiti, M., Si, Z., Trenchea, C., Partitioned penalty methods for the transport equation in the evolutionary Stokes-Darcy-transport problem. Numer. Methods PDEs., 35 (2019), no. 1, 349-374.

Gatica, G.N., Ruiz-BAier, R., Tierra, G., A mixed finite element methos for Darcy's equations with pressure dependent porosity. Math. Comput., 85 (2016), 1-33.

Jena, S.K., Mahpatra, S.K., SARKAR, A., Thermosolutal convection in a fluid-porous composite medium. Heat Transf. Asian Res., 42 (2013), no. 4, 281-299.

Joodi, A.S., Sizaret, S., Binet, S., Bruand, A., Alberic, P., Lepiller, M., Development of a Darcy-Brinkman model to simulate water flow and tracer transport in a heterogeneous karstic aquifer (Val d'Orléans, France). Hydrogeology J., 18 (2010), 295-309.

Khaled, A.R.A., VAfAI, K., The role of porous media in modeling flow and heat transfer in biological tissues. Int. J. Heat Mass Transf., 46 (2003), 4989-5003.

Pontrelli, G., Blood flow through an axisymmetric stenosis. Proc. Int. Mech. Engrs., 215 (2001) H01-H10.

Quarteroni, A., Valli, A., Numerical Approximation of Partial Differential Equations. Springer Series in Computational Mathematics, vol. 23, Springer-Verlag Berlin Heidelberg, 1994. 
RiAz, A., Hesse, M., TChelePI, H.A., Onset of convection in gravitationally unstable diffusive boundary layer in porous media. J. Fluid Mech., 548 (2006) 87-111.

RUI, H., ZHANG, J., A stabilized mixed finite element method for coupled Stokes and Darcy flows with transport. Comput. Methods Appl. Mech. Engrg., 315 (2017), 169-189.

SPEZIALE, C.G., On the advantages of the vorticity-velocity formulations of the equations of fluid dynamics. J. Comput. Phys., 73 (1987), no. 2, 476-480.

Vassilev, D., Yotov, I., Coupling Stokes-Darcy flow with transport. SIAM J. Sci. Comput., 31 (2009), no. $5,3661-3684$.

VASSILEVski, P., Villa, U., A mixed formulation for the Brinkman problem. SIAM J. Numer. Anal., 52 (2014), no. 1, 258-281.

Zhang, J., RUI, H., CAO, Y., A partitioned method with different time steps for coupled Stokes and Darcy flows with transport. Int. J. Numer. Anal. Model., 15 (2019), no. 3, 463-498. 